\title{
Genus distributions of graphs under self-edge-amalgamations
}

\author{
Mehvish I. Poshni , Imran F. Khan , Jonathan L. Gross \\ Columbia University, Department of Computer Science NY 10027 USA, New York, USA
}

Received 19 July 2010, accepted 29 July 2011, published online 12 January 2012

\begin{abstract}
We investigate the well-known problem of counting graph imbeddings on all oriented surfaces with a focus on graphs that are obtained by pasting together two root-edges of another base graph. We require that the partitioned genus distribution of the base graph with respect to these root-edges be known and that both root-edges have two 2-valent endpoints. We derive general formulas for calculating the genus distributions of graphs that can be obtained either by self-co-amalgamating or by self-contra-amalgamating a base graph whose partitioned genus distribution is already known. We see how these general formulas provide a unified approach to calculating genus distributions of many new graph families, such as co-pasted and contra-pasted closed chains of copies of the triangular prism graph, as well as graph families like circular and Möbius ladders with previously known solutions to the genus distribution problem.
\end{abstract}

Keywords: Graph, genus distribution, self-edge-amalgamation.

Math. Subj. Class.: 05C10

\section{Introduction}

Prior research on the problem of counting oriented imbeddings of graphs on all 2-manifolds has yielded closed formulas, recursions for generating tabular data, and asymptotic lower bounds for various families of graphs. Our first installment [22] demonstrated a method for calculating partitioned genus distributions of arbitrarily long edge-linked chains obtained by iteratively edge-amalgamating copies of one or more base graphs of known partitioned genus distribution. In this installment, we develop two formulas that use the partitioned genus distribution of a graph to calculate the genus distribution of the graph obtained through self-edge-amalgamation of its two roots. Thus, combined with the results derived

E-mail addresses: poshni@cs.columbia.edu (Mehvish I. Poshni), imran@cs.columbia.edu (Imran F. Khan), gross@cs.columbia.edu (Jonathan L. Gross) 
in the first installment, we can first obtain a recursion for the genus distributions of an infinite family of open chains of edge-amalgamated copies of a base graph, and then apply the two formulas derived in this paper to obtain genus distributions of the corresponding one or two infinite families of closed chains. In this manner, one can calculate the genus distribution for arbitrarily large graphs. This paper is self-contained, though we strongly recommend reading [22] before reading this paper.

Precedents for work on counting imbeddings on various orientable and non-orientable surfaces include [2], [3], [4], [10], [12], [16], [17], [18], [20], [21], [24], [25], [26], [27], [28], [29], and [30] amongst many others. Some recent endeavors in this context have resulted in a series of related works in [11], [14], [7], [8], [9] and [23]. Prior work on counting graph imbeddings in a minimum-genus surface includes [1], [5], [6], and [15]. We briefly review some of the terminology introduced in [22].

We define a double-edge-rooted graph as a graph that has two distinct edges designated as root-edges, or more simply, as roots. In this paper, we require that a root-edge have two 2 -valent endpoints. We use the notation $(G, e, f)$ to mean that $G$ is double-edge-rooted, with edges $e$ and $f$ serving as root-edges. We can abbreviate $(G, e, f)$ as $G$ where it is clear from context that a double-edge-rooted graph is intended. A closed walk traced just inside the boundary of a face of an embedding is referred to as a face-boundary walk. We abbreviate face-boundary walk as $\boldsymbol{f b}$-walk. We define a strand, with respect to a root-edge $e$ (e-strand for short), to be an open subwalk of an fb-walk that runs between any two occurrences of the endpoints of $e$, such that it has in its interior neither an occurrence of $e$ nor of the endpoints of $e$.

The remainder of this section reviews some concepts and terminology introduced in the first installment of this paper, followed by a formal description of the self-edge-amalgamation operation.

\section{First-order sub-partials}

For each imbedding of a double-edge-rooted graph, there are two possibilities for each of its root-edges. Either,

- two distinct fb-walks are incident on it;

- or the same fb-walk is incident on both sides of it.

Accordingly, we use the mnemonic $d$ for "different" and $s$ for "same", and we categorize each imbedding of the double-edge-rooted graph $(G, e, f)$ into the four types: $d d, d s, s d$, or $s s$. The first letter in each of these types is for root-edge $e$ and the second for the rootedge $f$.

The genus distribution of a double-edge-rooted graph $(G, e, f)$ is further partitioned into the double-root partials $d d_{i}(G, e, f), d s_{i}(G, e, f), s d_{i}(G, e, f), s s_{i}(G, e, f)$. These are the numbers of cellular imbeddings of $(G, e, f)$ on the surface $S_{i}$ having types $d d, d s$, $s d$, or $s s$, respectively. For the purpose of our derivations, these partials are refined into the following first-order sub-partials:

- $d d_{i}^{0}(G, e, f), d s_{i}^{0}(G, e, f), s d_{i}^{0}(G, e, f)$ and $s s_{i}^{0}(G, e, f)$ are the numbers of imbeddings of $G$ on surface $S_{i}$ of types $d d, d s, s d$ and $s s$, respectively, such that none of the $\mathrm{fb}$-walks incident on $e$ is incident on $f$.

- $d d_{i}^{\prime}(G, e, f), d s_{i}^{\prime}(G, e, f)$, and $s d_{i}^{\prime}(G, e, f)$ are the numbers of imbeddings of $G$ on surface $S_{i}$ of types $d d, d s$ and $s d$, respectively, such that exactly one fb-walk incident on $e$ is also incident on $f$. 
- $d d_{i}^{\prime \prime}(G, e, f)$ is the number of imbeddings of $G$ on surface $S_{i}$ of type $d d$ such that both fb-walks incident on $e$ are also incident on $f$.

- $s s_{i}^{1}(G, e, f)$ and $s s_{i}^{2}(G, e, f)$ partition the number of imbeddings on surface $S_{i}$ of type $s s$ which are not of sub-type $s s^{0}$. The sub-partial $s s^{1}$ counts the cases where exactly one $e$-strand contains both occurrences of $f$, while the sub-partial $s s^{2}$ counts the cases where each $e$-strand contains an occurrence of $f$.

It follows from these definitions that each double-root partial is the sum of its first-order sub-partials. Moreover,

$$
g_{i}(G)=d d_{i}(G)+d s_{i}(G)+s d_{i}(G)+s s_{i}(G)
$$

\section{Second-order sub-partials}

The four first-order sub-partials $d d^{\prime}, d d^{\prime \prime}, d s^{\prime}$ and $s d^{\prime}$ are further refined into second-order sub-partials. To define these, we imagine assigning arbitrary orientations to root-edges $e$ and $f$ of the graph $(G, e, f)$. These assigned orientations are referred to as pastingorientations of root-edges. Given an oriented imbedding of $(G, e, f)$, as we walk along the oriented root-edge $e$ towards its head, the left side of the edge is labeled 1 and the right side is labeled 2. Whereas when we walk along root-edge $f$ towards its head, the left side is labeled 3 and the right side is labeled 4. This is illustrated in Figure 1.

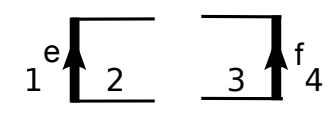

Figure 1: Model for second-order sub-partials with labelled edge-sides.

Accordingly, the second-order partials are described as follows:

- By definition, a $d d^{\prime}$-type imbedding has exactly one fb-walk incident on both rootedges. However, the fb-walk incident on both root-edges may combine either the faces 1 and 4, the faces 2 and 3, the faces 1 and 3, or the faces 2 and 4 . Accordingly, we define ${\overline{d d^{\prime}}}_{i}(G, e, f),{\widetilde{d d^{\prime}}}_{i}(G, e, f),{\overrightarrow{d d^{\prime}}}_{i}(G, e, f)$ and ${\overleftarrow{d d^{\prime}}}_{i}(G, e, f)$ to be the numbers of imbeddings of type $d d_{i}^{\prime}$ such that $(1,4),(2,3),(1,3)$ and $(2,4)$ are the respective pairs of sides that occur on the same fb-walk, as shown in the top row of Figure 2.

- ${\overrightarrow{d d^{\prime \prime}}}_{i}(G, e, f)$ is the number of imbeddings of type $d d_{i}^{\prime \prime}$ such that the pair of sides $(1,4)$ occurs on one of the fb-walks incident on the root-edges $e$ and $f$ and the pair of sides $(2,3)$ occurs on the other. Also, ${\overleftarrow{d d^{\prime \prime}}}_{i}(G, e, f)$ is defined similarly, but with $(1,3)$ and $(2,4)$ as pairs of sides occurring on the two fb-walks incident on root-edges $e$ and $f$.

- ${\overrightarrow{d s^{\prime}}}_{i}(G, e, f)$ and $\overleftarrow{d s^{\prime}}{ }_{i}(G, e, f)$ are the numbers of imbeddings of type $d s^{\prime}$ such that the three sides 1,3,4 (in the former case) and the three sides 2,3,4 (in the latter case) all occur on the same fb-walk.

- ${\overrightarrow{s d^{\prime}}}_{i}(G, e, f)$ and $\overleftarrow{s d^{\prime}}{ }_{i}(G, e, f)$ are the numbers of imbeddings of type $s d^{\prime}$ such that the three sides 1,2,4 (in the former case) and the three sides 1,2,3 (in the latter case) all occur in the same fb-walk. 
Clearly, each first-order sub-partial is the sum of its second-order sub-partials. A partitioned genus distribution of a graph is its genus distribution partitioned into sub-partials.

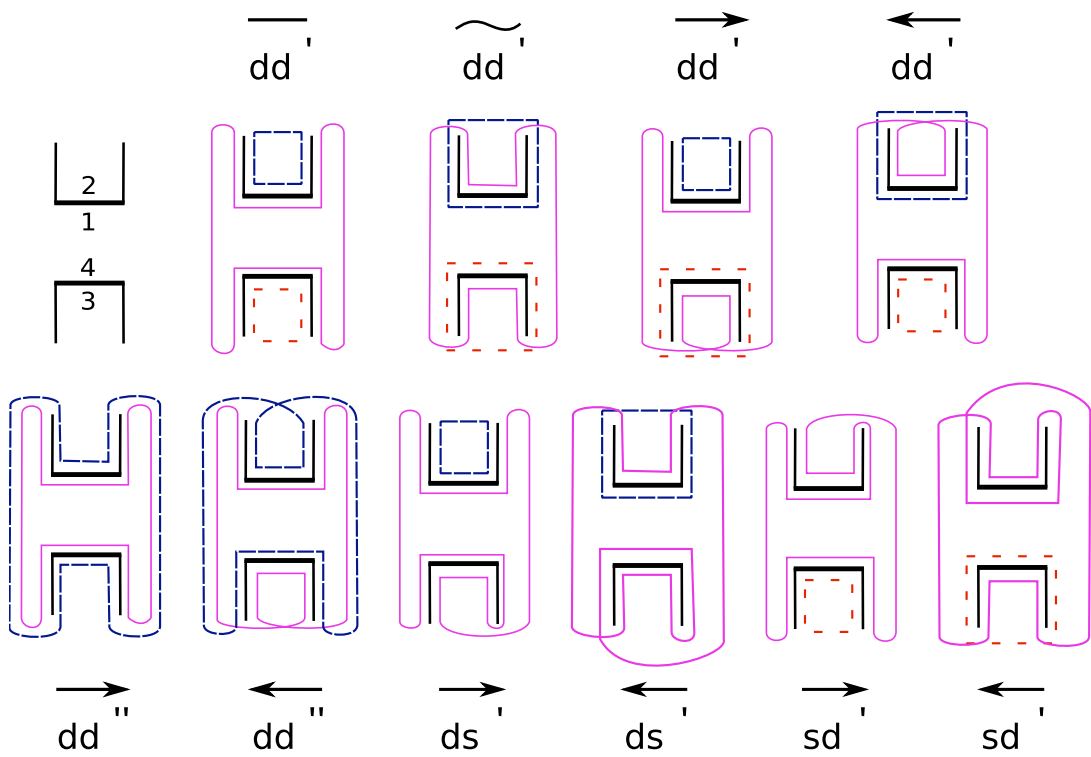

Figure 2: Modeling second-order sub-partials.

\section{Self-edge-amalgamation}

The self-edge-amalgamation of a graph is an operation whereby two root-edges of a double-edge-rooted graph are pasted together. Amongst many other classes, one can obtain families of closed chains of graphs, including circular ladders and Möbius ladders, by applying self-edge-amalgamation to families of open chains, such as the closed-end ladders. Informally, we use pasting to refer to any kind of amalgamation. Where it is clear from context that a self-edge-amalgamation is intended, we may use the terminology self-amalgamation. We denote this operation by a monadic operator acting on a doubleedge-rooted graph operand $(G, e, f)$ :

$$
W=*_{e f}(G, e, f)
$$

The self-edge-amalgamation of a double-edge-rooted graph $(G, e, f)$ produces a graph $W$ obtained from $G$ by identifying edges $e$ and $f$. We fix the pasting-orientations on the root-edges by arbitrarily orienting $e$ and $f$. Accordingly, the edge-ends of $e$ and $f$ at the tail are $e^{-}$and $f^{-}$, while the ones at the head are $e^{+}$and $f^{+}$. Now edges $e$ and $f$ can be pasted in two different ways. One way of pasting, called co-self-amalgamation, identifies the edge-end $e^{-}$with $f^{-}$and the edge-end $e^{+}$with $f^{+}$. The other way of pasting, called contra-self-amalgamation, pairs the edge-end $e^{-}$with $f^{+}$and the edge-end $e^{+}$with $f^{-}$. These two ways of self-edge-amalgamating a graph produces graphs which may be nonisomorphic, as seen later in this paper.

REMARK Assignment of labels 1 through 4 to the edge-sides of root-edges is also relative to these same pasting-orientations. 
We defined open chains in [22] as graphs formed by iteratively pasting copies of smaller graphs along root-edges. Graphs that are obtained from a self-amalgamation of double-edge-rooted open chains are referred to as closed chains. Depending on which type of pasting is used, these may be classified as co-pasted or contra-pasted closed chains.

We work under the assumption that we already have the partitioned genus distribution of the graph that we wish to self-edge-amalgamate. For smaller graphs, this can be done easily by using the Heffter-Edmonds algorithm [13]. For large graphs, some new ways of finding partitioned genus distributions are presented in [11], [14] and [22].

\section{Productions for self-edge-amalgamation}

Let $x_{i}$ be any double-root sub-partial. Then a production is used to represent the ways in which an imbedding of a double-edge-rooted graph $(G, e, f)$ of type $x$ on surface $S_{i}$ self-edge-amalgamates on the root-edges $e$ and $f$ to give various types of imbeddings of the resulting graph $W$. Formally, we write

$$
x_{i}(G, e, f) \longrightarrow g_{k_{1}}(W)+g_{k_{2}}(W)+g_{k_{3}}(W)+g_{k_{4}}(W)
$$

where $k_{1}, k_{2}, k_{3}, k_{4}$ are (not necessarily distinct) integer-valued functions of $i$. This can be read as follows:

An imbedding of $(G, e, f)$ of type $x$ on surface $S_{i}$ self-amalgamates on the root-edges $e$ and $f$ to give four imbeddings of the graph $W$ on the surfaces $S_{k_{1}}, S_{k_{2}}, S_{k_{3}}$ and $S_{k_{4}}$.

The left-hand side of the production is known as the production head and the right-hand side of the production as the production body.

REMARK The number of terms in the production body could be larger if the degrees of the endpoints of root-edges were larger.

The production, as we have defined it, does not specify whether the self-amalgamation is a co-self-paste or a contra-self-paste. However, as we shall see, for an application that seeks to find the genus distribution of a self-amalgamated graph, the system of productions will consistently refer to only one of the two types of self-pasting. While considering the self-edge-amalgamation for an imbedding on an oriented surface and modeling it using a production, it is important to maintain a sense of orientation of the strands. Each imbedding of a self-edge-amalgamated graph $W=*_{e f}(G, e, f)$ induces a unique imbedding of the graph $G$, such that the rotation system of $W$ is consistent with the rotation system of $G$.

Theorem 2.1. Let $(G, e, f)$ be a double-edge-rooted graph, where both root-edges have two 2-valent endpoints. Then the following productions apply to all scenarios of co-selfpaste and contra-self-paste in which no fb-walk of the imbedding of $G$ is incident on both root-edges $e$ and $f$ :

$$
\begin{aligned}
d d_{i}^{0}(G) & \longrightarrow 2 g_{i+1}(W)+2 g_{i+2}(W) \\
d s_{i}^{0}(G) & \longrightarrow 4 g_{i+1}(W) \\
s d_{i}^{0}(G) & \longrightarrow 4 g_{i+1}(W) \\
s s_{i}^{0}(G) & \longrightarrow 4 g_{i+1}(W)
\end{aligned}
$$


Proof. The proof of Production (2.1) follows by face-tracing of the fb-walks incident on both root-edges of the graph $G$. We examine in closer detail the recombination of strands caused by a self-edge-amalgamation where the imbedding of graph $G$ is of type $d d^{0}$. The production shown in the upper half of Figure 3 depicts the case of a co-self-paste while the drawing in the lower half shows a contra-self-paste on the same root-edges. In both cases we get the same results.
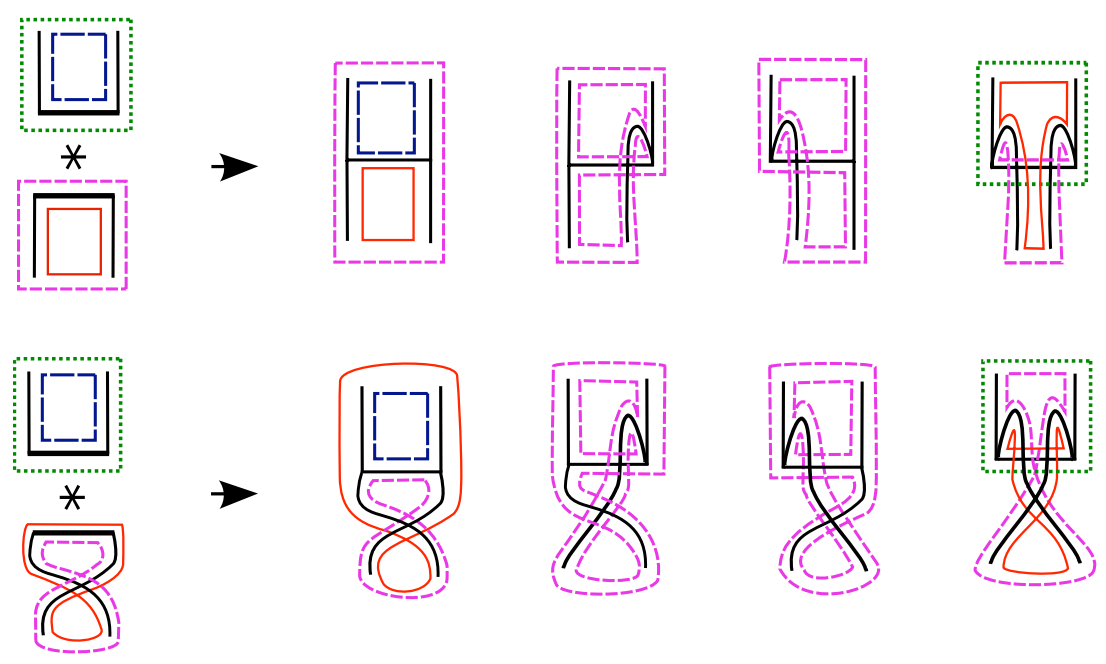

Figure 3: Productions for co-self-pasting and contra-self-pasting a $d d^{0}$-type imbedding of $(G, e, f)$.

The self-amalgamation produces two fewer vertices and one less edge in each of the four resultant graph imbeddings. The first and last imbeddings shown for each production have two fb-walks merging as a consequence of self-amalgamation. In the second and third imbeddings, all four fb-walks that are incident on the two root-edges merge into a single fbwalk. Applying the Euler polyhedral equation, we see that in the former case the decrease of a single face results in a genus increment of 1 , while in the latter case the decrease of three $\mathrm{fb}$-walks results in a genus increment of 2 . The proofs for the remaining productions are similar and also follow by face-tracing. For the sake of brevity, we leave these to the reader.

For imbeddings in which one or two fb-walks are incident on both root-edges, the productions for co-self-pasting and contra-self-pasting may differ. In particular, for $d d^{\prime}$ and $d d^{\prime \prime}$ we get different results for both ways of pasting.

Theorem 2.2. Let $(G, e, f)$ be a double-edge-rooted graph, where both root-edges have two 2-valent endpoints. Then the following productions describe all cases of co-self-pasting for imbeddings of $G$ of type $d d^{\prime}$ :

$$
\begin{aligned}
& {\overline{d d^{\prime}}}_{i}(G) \longrightarrow g_{i}(W)+3 g_{i+1}(W) \\
& {\widetilde{d d^{\prime}}}_{i}(G) \longrightarrow g_{i}(W)+3 g_{i+1}(W)
\end{aligned}
$$




$$
\begin{aligned}
& {\overrightarrow{d d^{\prime}}}_{i}(G) \longrightarrow 4 g_{i+1}(W) \\
& {\overleftarrow{d d^{\prime}}}_{i}(G) \longrightarrow 4 g_{i+1}(W)
\end{aligned}
$$

Furthermore, the following productions describe all cases of co-self-pasting for imbeddings of $G$ of type $d d^{\prime \prime}$ :

$$
\begin{aligned}
& {\overrightarrow{d d^{\prime \prime}}}_{i}(G) \longrightarrow 4 g_{i}(W) \\
& \overleftrightarrow{d d^{\prime \prime}}{ }_{i}(G) \longrightarrow 2 g_{i}(W)+2 g_{i+1}(W)
\end{aligned}
$$

Proof. For illustration of Production (2.5), which describes the effects of co-self-pasting a $\overline{d d^{\prime}}$-type imbedding of $G$, we refer to the upper half of Figure 4 . We observe that the first imbedding of graph $W$ stands out from the other three, in that there is a net increase of one fb-walk as the fb-walk incident on both roots of the imbedding of $G$ breaks into two $\mathrm{fb}$-walks during self-pasting. This does not occur in the other three cases, where there is a net decrease of one fb-walk in the resulting imbedding. The former results in an unchanged genus of the resultant graph imbedding, while the latter results in a genus increment of one. This accounts for Production (2.5).
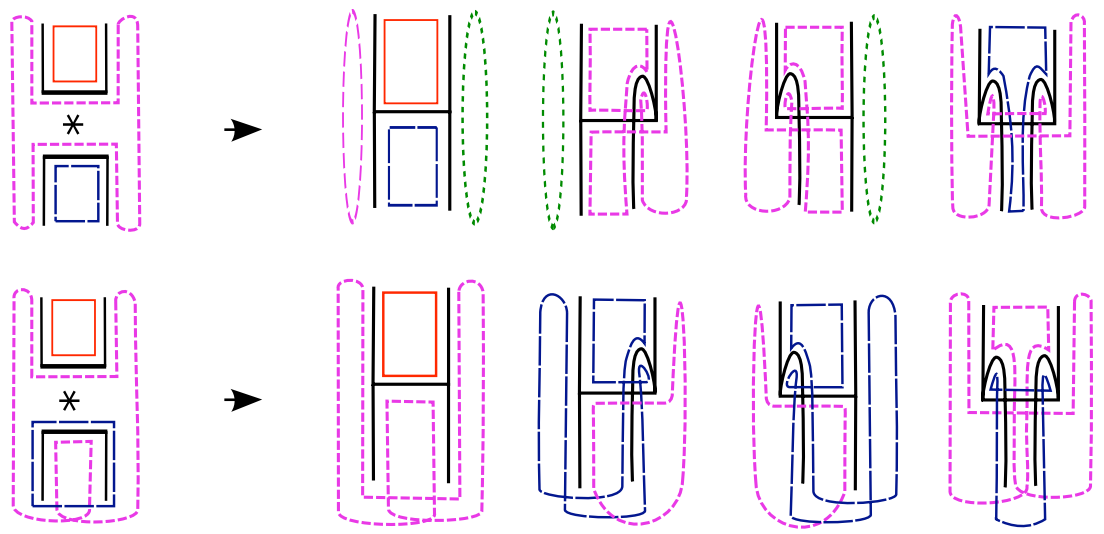

Figure 4: Productions for co-self-pasting $\overline{d d^{\prime}}$-type and $\overrightarrow{d d^{\prime}}$-type imbeddings of $(G, e, f)$.

For Production (2.7), the illustration in the bottom half of Figure 4 shows that all four imbeddings resulting from the self-pasting of $G$ end up with one less face, thereby warranting a genus increment of 1 for the resulting imbeddings. Proofs for Productions (2.6) and (2.8) are similar to the proofs for (2.5) and (2.7), respectively, and we leave them to the reader.

Productions (2.9) and (2.10) for co-self-pasting a $\overrightarrow{d d^{\prime \prime}}$ - or a $\overleftarrow{d d^{\prime \prime}}$-type imbedding of $G$ can also be derived by using the same technique. We include Figure 5 for aiding the reader with the proof of Production (2.10), and omit the proof of Production (2.9). 

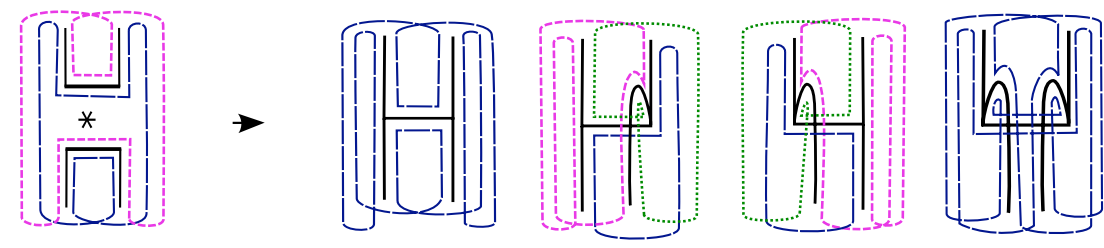

Figure 5: Production for co-self-pasting a $\overleftarrow{d d^{\prime \prime}}$-type imbedding of $(G, e, f)$

Theorem 2.3. Let $(G, e, f)$ be a double-edge-rooted graph, where both root-edges have two 2-valent endpoints. Then the following productions apply for contra-self-pasting all $d d^{\prime}$-type imbeddings of $G$ :

$$
\begin{aligned}
& {\overline{d d^{\prime}}}_{i}(G) \longrightarrow 4 g_{i+1}(W) \\
& {\widetilde{d d^{\prime}}}_{i}(G) \longrightarrow 4 g_{i+1}(W) \\
& {\overrightarrow{d d^{\prime}}}_{i}(G) \longrightarrow g_{i}(W)+3 g_{i+1}(W) \\
& {\overleftarrow{d d^{\prime}}}_{i}(G) \longrightarrow g_{i}(W)+3 g_{i+1}(W)
\end{aligned}
$$

Furthermore, the following productions apply for contra-self-pasting all dd"-type imbeddings of $G$ :

$$
\begin{aligned}
& {\overrightarrow{d d^{\prime \prime}}}_{i}(G) \longrightarrow 2 g_{i}(W)+2 g_{i+1}(W) \\
& {\overleftarrow{d d^{\prime \prime}}}_{i}(G) \longrightarrow 4 g_{i}(W)
\end{aligned}
$$

Proof. Figure 6 illustrates Production (2.12) in the upper half and Production (2.14) in the bottom half.

For Production (2.12), in all four imbeddings resulting from the contra-self-pasting, the three fb-walks incident on the root-edges of the graph $G$ break into strands that merge into two fb-walks, as shown in Figure 6. While, for Production (2.14), this happens for only three of the resulting imbeddings of graph $W$. For the remaining imbedding, the fb-walks break into strands that recombine to give four distinct fb-walks. Proofs of Productions (2.11) and (2.13) are similar and left to the reader.

Similarly, applying contra-self-pasting to the root-edges of a $\overrightarrow{d d^{\prime \prime}}$-type imbedding of $(G, e, f)$ results in two imbeddings of $W$ where all the fb-walks incident on the roots merge into a single fb-walk, and two imbeddings where they break into strands that recombine into three distinct fb-walks, as shown in Figure 7. This proves Production (2.15). Proof for Production (2.16) is similar and we leave it to the reader. 

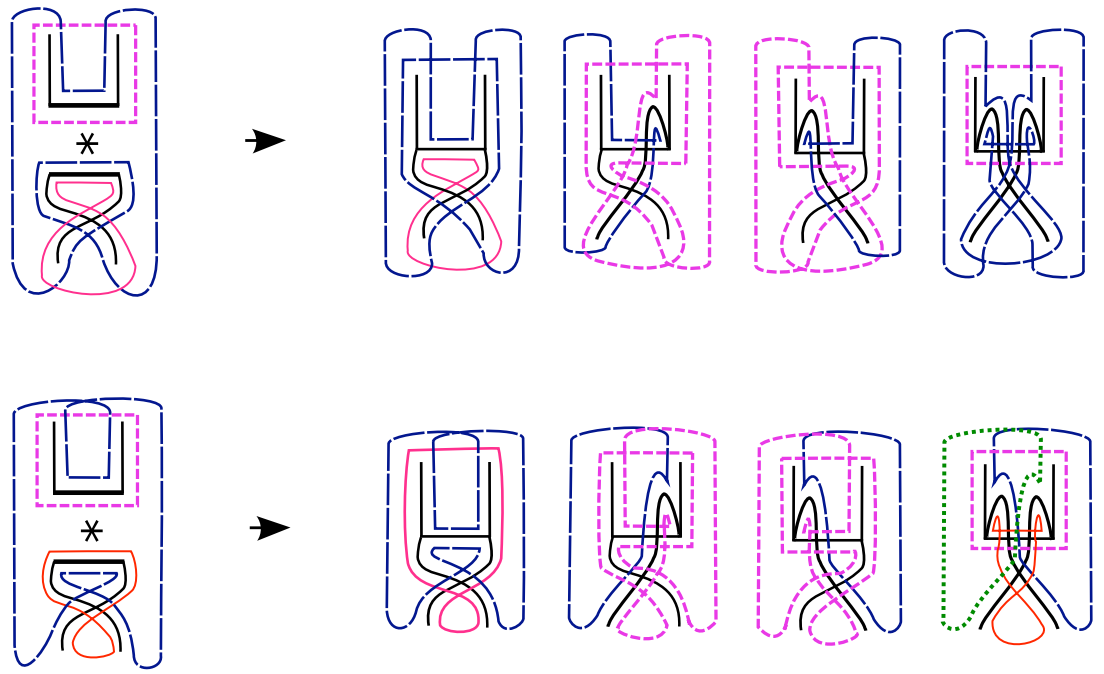

Figure 6: Productions for contra-self-pasting $\widetilde{d d^{\prime}}$-type and $\overleftarrow{d d^{\prime}}$-type imbeddings of $(G, e, f)$.
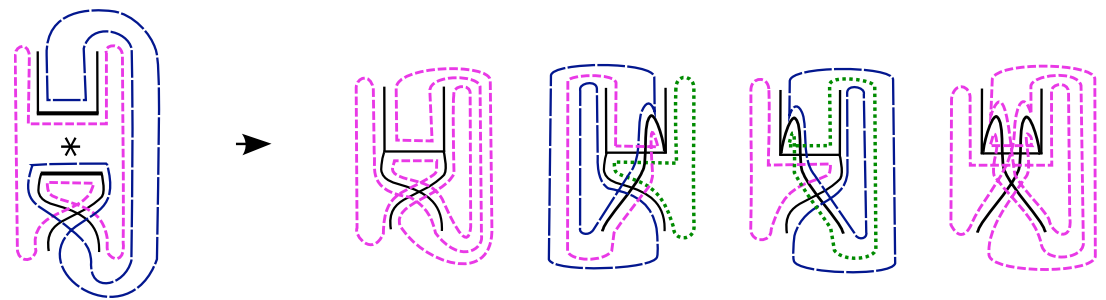

Figure 7: Production for contra-self-pasting $\overrightarrow{d d^{\prime \prime}}$-type imbeddings of $(G, e, f)$.

Theorem 2.4. Let $(G, e, f)$ be a double-edge-rooted graph, where both roots have two 2-valent endpoints. Then the following productions cover all scenarios for co-self-pasting and contra-self-pasting, where the imbedding of $G$ is of type $d s^{\prime}$ or $s d^{\prime}$ :

$$
\begin{aligned}
& d s_{i}^{\prime}(G) \quad \longrightarrow 2 g_{i}(W)+2 g_{i+1}(W) \\
& s d_{i}^{\prime}(G) \longrightarrow 2 g_{i}(W)+2 g_{i+1}(W)
\end{aligned}
$$

Proof. Due to the symmetry of the models $\overrightarrow{d s^{\prime}}$ and $\overrightarrow{s d^{\prime}}$, and of $\overleftarrow{d s^{\prime}}$ and $\overleftarrow{s d^{\prime}}$, we need only provide the proof for one of the two Productions (2.17) and (2.18). Figure 8 illustrates the proof for the co-self-pasted and contra-self-pasted $\overrightarrow{d s^{\prime}}$-type imbedding of $G$. We get two imbeddings with a genus increment of 0 and two with a genus increment of 1 . We leave the proof for a $\overleftarrow{d s^{\prime}}$-type imbedding of $G$ to the reader. The reader will observe that the production body for the imbedding type $\overleftarrow{d s^{\prime}}$ is identical to the production body for the imbedding type $\overrightarrow{d s^{\prime}}$. This is true for both co-self-pasting and contra-self-pasting operations. This completes the proof of Production (2.17). 

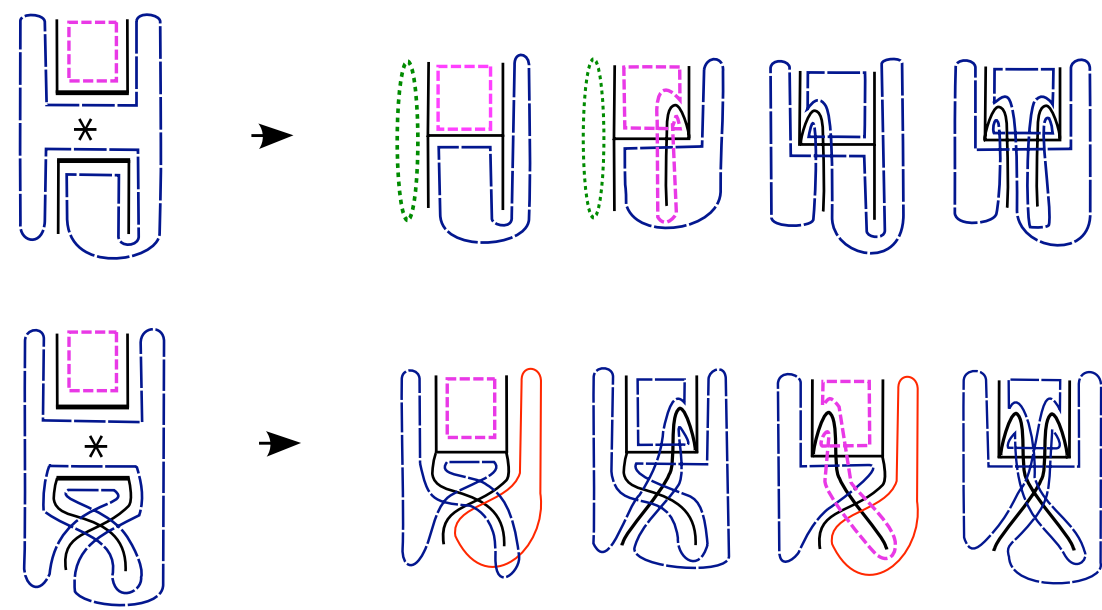

Figure 8: Productions for co-pasting and contra-pasting a $\overrightarrow{d s^{\prime}}$-type imbedding of $(G, e, d)$.

Theorem 2.5. Let $(G, e, f)$ be a double-edge-rooted graph, where both roots have two 2-valent endpoints. Then the following productions apply for co-self-pasting or contraself-pasting an $s s^{1}$ or $s s^{2}$-type imbedding of $G$ :

$$
\begin{aligned}
& s s_{i}^{1}(G) \longrightarrow 4 g_{i}(W) \\
& s s_{i}^{2}(G) \longrightarrow 3 g_{i}(W)+g_{i-1}(W)
\end{aligned}
$$

Proof. When the imbedding of a double-edge-rooted graph $G$ is of type $s s^{1}$, a self-amalgamation on the root-edges breaks the single fb-walk incident on both roots into strands that recombine to give two fb-walks in all the corresponding imbeddings of the self-amalgamated graph $W$. The additional face balances out the decrease of two vertices and one edge to retain the same genus in each of the four resulting imbeddings of $W$. This holds for co-self-pasting as well as contra-self-pasting as evident from Figure 9.

We leave the proof of Production 2.20 for the self-amalgamation of an $s s^{2}$-type imbedding to the reader.

REMARK As it turns out, the productions for second-order sub-partial types of $d d^{\prime}$ and $d d^{\prime \prime}$ are the only ones that disagree for a co-self-paste and a contra-self-paste. Moreover, the results for the co- and contra-self-amalgamation for both $d d^{\prime}$ - and $d d^{\prime \prime}$-type imbeddings are symmetric in the sense that the production body for co-self-pasting a $\overrightarrow{d d^{\prime \prime}}$-type (or a $\overleftarrow{d d^{\prime \prime}}$-type) imbedding of $G$ is the same as the production body for contra-self-pasting $\overleftarrow{d d^{\prime \prime}}$-type (or a $\overrightarrow{d d^{\prime \prime}}$-type) imbedding of $G$. Likewise, for the pairs of $\overline{d d^{\prime}}$-, $\widetilde{d d^{\prime}}$-types of imbeddings of $G$ which show a symmetry with the $\overrightarrow{d d^{\prime}}-, \overleftarrow{d d^{\prime}}$-types of imbeddings of $G$. 

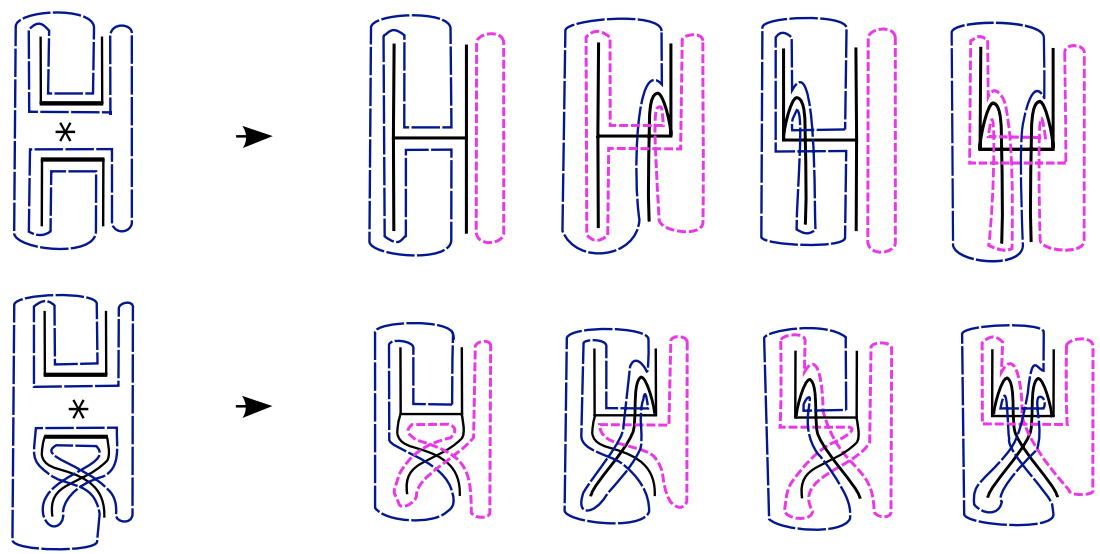

Figure 9: Productions for co-self-pasting and contra-self-pasting an $s s^{1}$-type imbedding of $(G, e, f)$.

Theorem 2.6. Let $W$ be the graph formed by co-self-pasting of $(G, e, f)$. Then,

$$
\begin{aligned}
g_{i}(W)= & 2 d d_{i-2}^{0}(G)+2 d d_{i-1}^{0}(G)+3{\overline{d d^{\prime}}}_{i-1}(G)+3{\widetilde{d d^{\prime}}}_{i-1}(G)+4{\overrightarrow{d d^{\prime}}}_{i-1}(G) \\
& +4 \overleftarrow{d d^{\prime}}{ }_{i-1}(G)+2 \overleftarrow{d d^{\prime \prime}}{ }_{i-1}(G)+4 d s_{i-1}^{0}(G)+2 d s_{i-1}^{\prime}(G)+4 s d_{i-1}^{0}(G) \\
& +2 s d_{i-1}^{\prime}(G)+4 s s_{i-1}^{0}(G)+{\overline{d d^{\prime}}}_{i}(G)+{\widetilde{d d^{\prime}}}_{i}(G)+4{\overrightarrow{d d^{\prime \prime}}}_{i}(G)+2{\overleftarrow{d d^{\prime \prime}}}_{i}(G) \\
& +2 d s_{i}^{\prime}(G)+2 s d_{i}^{\prime}(G)+4 s s_{i}^{1}(G)+3 s s_{i}^{2}(G)+s s_{i+1}^{2}(G)
\end{aligned}
$$

Proof. The Production (2.1):

$$
d d_{i}^{0}(G) \longrightarrow 2 g_{i+1}(W)+2 g_{i+2}(W)
$$

indicates that each $d d^{0}$-type imbedding of $(G, e, f)$ on the surface $S_{i}$ when self-amalgamated, induces two imbeddings of $W$ on $S_{i+1}$ and two on the surface $S_{i+2}$. These contributions account for the first two terms $2 d d_{i-2}^{0}(G)+2 d d_{i-1}^{0}(G)$ on the right-hand side of Equation (2.21). Taking into account all the contributions made by productions listed in Theorems 2.1-2.5, the result follows.

Theorem 2.7. Let $W$ be the graph formed by contra-self-pasting of $(G, e, f)$. Then,

$$
\begin{aligned}
g_{i}(W)= & 2 d d_{i-2}^{0}(G)+2 d d_{i-1}^{0}(G)+3{\overrightarrow{d d^{\prime}}}_{i-1}(G)+3{\overleftarrow{d d^{\prime}}}_{i-1}(G)+4{\overrightarrow{d d^{\prime}}}_{i-1}(G) \\
& +4{\widetilde{d d^{\prime}}}_{i-1}(G)+2{\overrightarrow{d d^{\prime \prime}}}_{i-1}(G)+4 d s_{i-1}^{0}(G)+2 d s_{i-1}^{\prime}(G)+4 s d_{i-1}^{0}(G) \\
& +2 s d_{i-1}^{\prime}(G)+4 s s_{i-1}^{0}(G)+{\overrightarrow{d d^{\prime}}}_{i}(G)+{\overleftarrow{d d^{\prime}}}_{i}(G)+4{\overleftarrow{d d^{\prime \prime}}}_{i}(G)+2{\overrightarrow{d d^{\prime \prime}}}_{i}(G) \\
& +2 d s_{i}^{\prime}(G)+2 s d_{i}^{\prime}(G)+4 s s_{i}^{1}(G)+3 s s_{i}^{2}(G)+s s_{i+1}^{2}(G)
\end{aligned}
$$

Proof. The proof for Equation (2.22) is obvious from Equation (2.21) and our earlier remarks on the symmetries of the productions for $d d^{\prime}$ and $d d^{\prime \prime}$ second-order sub-partial types. 
Thus, depending on whether one plans on forming a closed chain through a co-selfamalgamation or through a contra-self-amalgamation, the genus distribution of the closed chain is calculated by using Theorems 2.6 or 2.7, respectively. For instance, using the partitioned genus distributions calculated for closed-end ladders in [22], we can now use Theorems 2.6 and 2.7 to obtain partitioned genus-distribution for both circular and Möbius ladders.

\section{Application: Revisiting circular ladders and Möbius ladders}

The genus distributions of circular ladders and Möbius ladders were first derived by [19]. $\S 4$ of [22] shows how calculation of the double-root genus distributions of closed-end ladders is reducible to a routine recursion. This in turn reduces the derivation of the genus distributions of circular and Möbius ladders, in turn, to a routine substitution into an equation.

Let $L_{n}$ be the closed-end ladder with 2 end-rungs and $n$ interior rungs, as shown in Figure 10.

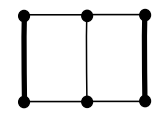

$\mathrm{L}_{1}$

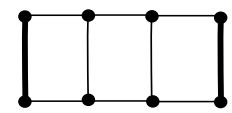

$\mathrm{L}_{2}$

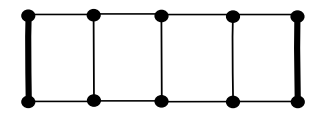

$\mathrm{L}_{3}$

Figure 10: Closed-end ladders $L_{n}$.

Let $C L_{n}$ denote the circular ladder with $n$ rungs as illustrated in Figure 11. We observe that co-self-pasting the closed-end ladder $L_{n}$ on the root-edges yields $C L_{n+1}$. Similarly,
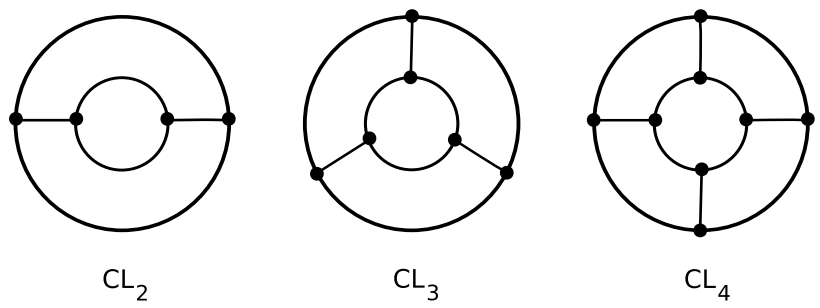

Figure 11: Circular ladders $C L_{n}$.

we denote a Möbius ladder with $n$ rungs by $M L_{n}$ as shown in Figure 12. It can be observed that contra-self-pasting the closed-end ladder $L_{n}$ on the root-edges yields $M L_{n+1}$.

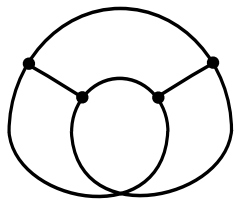

$\mathrm{ML}_{2}$

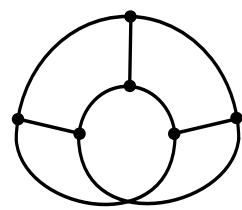

$\mathrm{ML}_{3}$

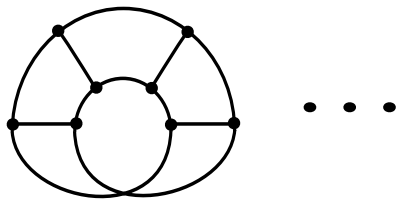

$\mathrm{ML}_{4}$

Figure 12: Möbius ladders $M L_{n}$. 
We give a small example that demonstrates how to calculate genus distributions of $C L_{4}$ and $M L_{4}$ from the partitioned genus distribution of $L_{3}$. We begin by reproducing in Table 1 the partitioned genus distribution of the closed-end ladder $L_{3}$ originally derived in [22].

Table 1: Double-root partials of $L_{3}$.

\begin{tabular}{|c|ccc||c|ccc|}
\hline \hline \multicolumn{7}{|c|}{$L_{3}$} \\
\hline$k$ & 0 & 1 & 2 & $k$ & 0 & 1 & 2 \\
\hline$d d_{k}^{0}$ & 6 & 0 & 0 & ${\overrightarrow{d s^{\prime}}}_{k}$ & 0 & 2 & 0 \\
${\overrightarrow{d d^{\prime}}}_{k}$ & 1 & 6 & 0 & ${\overleftarrow{d s^{\prime}}}_{k}$ & 0 & 2 & 0 \\
${\widetilde{d d^{\prime}}}_{k}$ & 1 & 6 & 0 & $s d_{k}^{0}$ & 0 & 4 & 0 \\
${\overrightarrow{d d^{\prime}}}_{k}$ & 0 & 6 & 0 & ${\overrightarrow{s d^{\prime}}}_{k}$ & 0 & 2 & 0 \\
${\overleftarrow{d d^{\prime}}}_{k}$ & 0 & 6 & 0 & ${\overleftarrow{s d^{\prime}}}_{k}$ & 0 & 2 & 0 \\
${\overrightarrow{d d^{\prime \prime}}}_{k}$ & 0 & 0 & 0 & $s s_{k}^{0}$ & 0 & 0 & 0 \\
${\overleftarrow{d d^{\prime \prime}}}_{k}$ & 0 & 0 & 0 & $s s_{k}^{1}$ & 0 & 0 & 8 \\
$d s_{k}^{0}$ & 0 & 4 & 0 & $s s_{k}^{2}$ & 0 & 0 & 8 \\
\hline \hline
\end{tabular}

Simply plugging the values from Table 1 into Equation (2.21) yields the genus distributions of circular ladder $C L_{4}$.

$$
\begin{aligned}
g_{0}\left(C L_{4}\right)= & {\overline{d d^{\prime}}}_{0}\left(L_{3}\right)+\widetilde{d d^{\prime}}{ }_{0}\left(L_{3}\right)=1+1=2 \\
g_{1}\left(C L_{4}\right)= & 2 d d_{0}^{0}\left(L_{3}\right)+3 \overline{d d^{\prime}}{ }_{0}\left(L_{3}\right)+3 \widetilde{d d^{\prime}}{ }_{0}\left(L_{3}\right)+{\overline{d d^{\prime}}}_{1}\left(L_{3}\right)+\widetilde{d d^{\prime}}{ }_{1}\left(L_{3}\right)+2 d s_{1}^{\prime}\left(L_{3}\right) \\
& +2 s d_{1}^{\prime}\left(L_{3}\right)+s s_{2}^{2}\left(L_{3}\right) \\
= & 2 \times 6+3 \times 1+3 \times 1+6+6+2 \times 4+2 \times 4+8 \\
= & 54 \\
g_{2}\left(C L_{4}\right)= & 2 d d_{0}^{0}\left(L_{3}\right)+3{\overline{d d^{\prime}}}_{1}\left(L_{3}\right)+3{\widetilde{d d^{\prime}}}_{1}\left(L_{3}\right)+4{\overrightarrow{d d^{\prime}}}_{1}\left(L_{3}\right)+4{\overleftarrow{d d^{\prime}}}_{1}\left(L_{3}\right)+4 d s_{1}^{0}\left(L_{3}\right) \\
& +2 d s_{1}^{\prime}\left(L_{3}\right)+4 s d_{1}^{0}\left(L_{3}\right)+2 s d_{1}^{\prime}\left(L_{3}\right)+4 s s_{2}^{1}\left(L_{3}\right)+3 s s_{2}^{2}\left(L_{3}\right) \\
= & 2 \times 6+3 \times 6+3 \times 6+4 \times 6+4 \times 6+4 \times 4+2 \times 4+4 \times 4 \\
& +2 \times 4+4 \times 8+3 \times 8 \\
= & 200 \\
g_{3}\left(C L_{4}\right)= & 0
\end{aligned}
$$


Whereas, plugging the values from Table 1 into Equation (2.22) produces the genus distribution of the Möbius ladder $M L_{4}$ :

$$
\begin{aligned}
g_{0}\left(M L_{4}\right)= & 0 \\
g_{1}\left(M L_{4}\right)= & 2 d d_{0}^{0}\left(L_{3}\right)+4{\overline{d d^{\prime}}}_{0}\left(L_{3}\right)+4{\widetilde{d d^{\prime}}}_{0}\left(L_{3}\right)+{\overrightarrow{d d^{\prime}}}_{1}\left(L_{3}\right)+{\overleftarrow{d d^{\prime}}}_{1}\left(L_{3}\right)+2 d s_{1}^{\prime}\left(L_{3}\right) \\
& +2 s d_{1}^{\prime}\left(L_{3}\right)+s s_{2}^{2}\left(L_{3}\right) \\
= & 2 \times 6+4 \times 1+4 \times 1+6+6+2 \times 4+2 \times 4+8 \\
= & 56 \\
g_{2}\left(M L_{4}\right)= & 2 d d_{0}^{0}\left(L_{3}\right)+3{\overrightarrow{d d^{\prime}}}_{1}\left(L_{3}\right)+3{\overleftarrow{d d^{\prime}}}_{1}\left(L_{3}\right)+4{\overline{d d^{\prime}}}_{1}\left(L_{3}\right)+4{\widetilde{d d^{\prime}}}_{1}\left(L_{3}\right) \\
& +4 d s_{1}^{0}\left(L_{3}\right)+2 d s_{1}^{\prime}\left(L_{3}\right)+4 s d_{1}^{0}\left(L_{3}\right)+2 s d_{1}^{\prime}\left(L_{3}\right)+4 s s_{2}^{1}\left(L_{3}\right)+3 s s_{2}^{2}\left(L_{3}\right) \\
= & 2 \times 6+3 \times 6+3 \times 6+4 \times 6+4 \times 6+4 \times 4+2 \times 4+4 \times 4+2 \times 4 \\
& +4 \times 8+3 \times 8 \\
= & 200 \\
g_{3}\left(M L_{4}\right)= & 0
\end{aligned}
$$

\section{Application: Closed chains of copies of a triangular prism graph}

As an example of two entirely new calculations of genus distributions of closed chains, we consider closed chains of copies of the triangular prism graph. Figure 13 shows a triangular prism graph at the left, where two of its edges are trisected and their middle-thirds are designated as root-edges. We show the root-edges darker by convention.

Let $\Delta_{G}$ denote the double-edge-rooted triangular prism graph. Figure 13 shows some small double-edge-rooted open chains of copies of the graph $\Delta_{G}$. We denote an open chain consisting of $n$ copies of $\Delta_{G}$ by $\mathcal{P} r_{n}$.
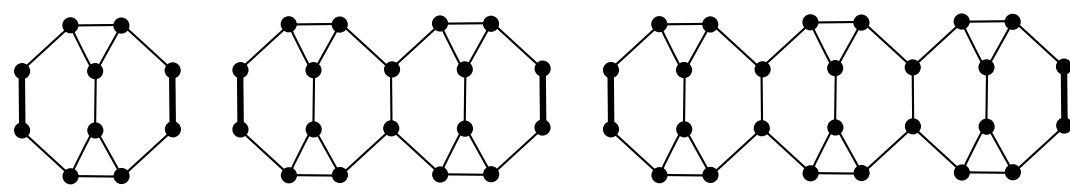

Figure 13: Open chains of copies of a triangular prism graph.

We begin the derivations for closed chains of copies of $\Delta_{G}$ by first producing the partitioned genus distribution of the open chains $\mathcal{P} r_{1}$ and $\mathcal{P} r_{2}$ in Table 2. These have been derived using the methods in [22] . 
Table 2: Genus distributions of the open chains $\mathcal{P} r_{1}$ and $\mathcal{P} r_{2}$ of 1 and 2 copies of $\Delta_{G}$, respectively.

\begin{tabular}{|c|rrr|rrrrr|}
\hline \hline $\mathcal{P} r_{n}$ & & $\mathcal{P} r_{1}$ & & \multicolumn{5}{c|}{$\mathcal{P} r_{2}$} \\
\hline$k$ & 0 & 1 & 2 & 0 & 1 & 2 & 3 & 4 \\
\hline$d d_{k}^{0}$ & 0 & 0 & 0 & 6 & 176 & 704 & 0 & 0 \\
${\overrightarrow{d d^{\prime}}}_{k}$ & 1 & 0 & 0 & 1 & 46 & 400 & 0 & 0 \\
${\widetilde{d d^{\prime}}}_{k}$ & 1 & 0 & 0 & 1 & 46 & 400 & 0 & 0 \\
${\overrightarrow{d d^{\prime}}}_{k}$ & 0 & 0 & 0 & 0 & 22 & 256 & 0 & 0 \\
$\overleftarrow{d d^{\prime}}{ }_{k}$ & 0 & 0 & 0 & 0 & 22 & 256 & 0 & 0 \\
${\overrightarrow{d d^{\prime \prime}}}_{k}$ & 0 & 12 & 0 & 0 & 0 & 48 & 0 & 0 \\
$\overleftrightarrow{d d^{\prime \prime}}{ }_{k}$ & 0 & 0 & 0 & 0 & 0 & 48 & 0 & 0 \\
$d s_{k}^{0}$ & 0 & 4 & 0 & 0 & 44 & 800 & 384 & 0 \\
${\overrightarrow{d s^{\prime}}}_{k}$ & 0 & 4 & 0 & 0 & 6 & 272 & 960 & 0 \\
${\overleftarrow{d s^{\prime}}}_{k}$ & 0 & 4 & 0 & 0 & 6 & 272 & 960 & 0 \\
$s d_{k}^{0}$ & 0 & 4 & 0 & 0 & 44 & 800 & 384 & 0 \\
${\overrightarrow{s d^{\prime}}}_{k}$ & 0 & 4 & 0 & 0 & 6 & 272 & 960 & 0 \\
$\overleftarrow{s d^{\prime}}{ }_{k}$ & 0 & 4 & 0 & 0 & 6 & 272 & 960 & 0 \\
$s s_{k}^{0}$ & 0 & 0 & 0 & 0 & 0 & 320 & 1888 & 0 \\
$s s_{k}^{1}$ & 0 & 0 & 24 & 0 & 0 & 72 & 1664 & 2304 \\
$s s_{k}^{2}$ & 0 & 2 & 0 & 0 & 0 & 8 & 288 & 0 \\
\hline$g_{k}$ & 2 & 38 & 24 & 8 & 424 & 5200 & 8448 & 2304 \\
\hline \hline
\end{tabular}

Let $\mathcal{C P} r_{n}$ be the co-self-amalgamated closed chain of $n$ copies of $\Delta_{G}$, as shown in Figure 14. We plug the values from Table 2 into Equation (2.21) to calculate genus distributions for $\mathcal{C} \mathcal{P} r_{1}$ and $\mathcal{C P} r_{2}$.
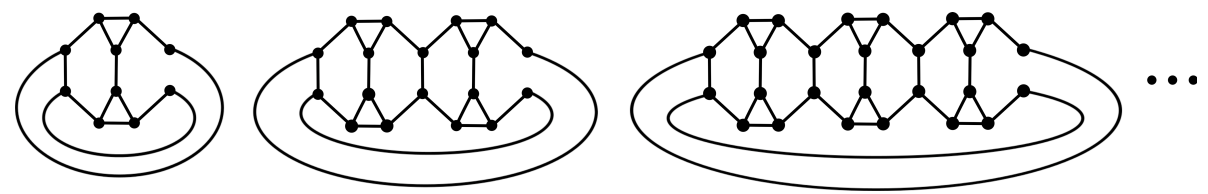

Figure 14: Co-pasted closed chains of copies of a triangular prism graph. 
We illustrate this as follows:

$$
\begin{aligned}
& g_{0}\left(\mathcal{C P} r_{1}\right)={\overline{d d^{\prime}}}_{0}\left(\mathcal{P} r_{1}\right)+\widetilde{d d^{\prime}}{ }_{0}\left(\mathcal{P} r_{1}\right)+4{\overrightarrow{d d^{\prime \prime}}}_{0}\left(\mathcal{P} r_{1}\right)+2 \overleftarrow{d d^{\prime \prime}}{ }_{0}\left(\mathcal{P} r_{1}\right)+2 d s_{0}^{\prime}\left(\mathcal{P} r_{1}\right) \\
& +2 s d_{0}^{\prime}\left(\mathcal{P} r_{1}\right)+4 s s_{0}^{1}\left(\mathcal{P} r_{1}\right)+3 s s_{0}^{2}\left(\mathcal{P} r_{1}\right)+s s_{1}^{2}\left(\mathcal{P} r_{1}\right) \\
& =1+1+4 \times 0+2 \times 0+2 \times 0+2 \times 0+4 \times 0+3 \times 0+2 \\
& =4 \\
& g_{1}\left(\mathcal{C P} r_{1}\right)=2 d d_{0}^{0}\left(\mathcal{P} r_{1}\right)+3 \overline{d d^{\prime}}{ }_{0}\left(\mathcal{P} r_{1}\right)+3 \widetilde{d d^{\prime}}{ }_{0}\left(\mathcal{P} r_{1}\right)+4{\overrightarrow{d d^{\prime}}}_{0}\left(\mathcal{P} r_{1}\right)+4 \overleftarrow{d d^{\prime}}{ }_{0}\left(\mathcal{P} r_{1}\right) \\
& +2 \overleftarrow{d d^{\prime \prime}}{ }_{0}\left(\mathcal{P} r_{1}\right)+4 d s_{0}^{0}\left(\mathcal{P} r_{1}\right)+2 d s_{0}^{\prime}\left(\mathcal{P} r_{1}\right)+4 s d_{0}^{0}\left(\mathcal{P} r_{1}\right)+2 s d_{0}^{\prime}\left(\mathcal{P} r_{1}\right) \\
& +4 s s_{0}^{0}\left(\mathcal{P} r_{1}\right)+\overline{d d^{\prime}}{ }_{1}\left(\mathcal{P} r_{1}\right)+\widetilde{d d^{\prime}}{ }_{1}\left(\mathcal{P} r_{1}\right)+4{\overrightarrow{d d^{\prime \prime}}}_{1}\left(\mathcal{P} r_{1}\right)+2 \overleftarrow{d d^{\prime \prime}}{ }_{1}\left(\mathcal{P} r_{1}\right) \\
& +2 d s_{1}^{\prime}\left(\mathcal{P} r_{1}\right)+2 s d_{1}^{\prime}\left(\mathcal{P} r_{1}\right)+4 s s_{1}^{1}\left(\mathcal{P} r_{1}\right)+3 s s_{1}^{2}\left(\mathcal{P} r_{1}\right)+s s_{2}^{2}\left(\mathcal{P} r_{1}\right) \\
& =2 \times 0+3 \times 1+3 \times 1+4 \times 0+4 \times 0+2 \times 0+4 \times 0+2 \times 0 \\
& +4 \times 0+2 \times 0+4 \times 0+0+0+4 \times 12+2 \times 0+2 \times 8+2 \times 8 \\
& +4 \times 0+3 \times 2+0 \\
& =92 \\
& g_{2}\left(\mathcal{C} \mathcal{P} r_{1}\right)=2 d d_{0}^{0}\left(\mathcal{P} r_{1}\right)+2 d d_{1}^{0}\left(\mathcal{P} r_{1}\right)+3 \overline{d d^{\prime}}{ }_{1}\left(\mathcal{P} r_{1}\right)+3 \widetilde{d d^{\prime}}{ }_{1}\left(\mathcal{P} r_{1}\right)+4 \overrightarrow{d d^{\prime}}{ }_{1}\left(\mathcal{P} r_{1}\right) \\
& +4 \overleftarrow{d d^{\prime}}{ }_{1}\left(\mathcal{P} r_{1}\right)+2 \overleftarrow{d d^{\prime \prime}}{ }_{1}\left(\mathcal{P} r_{1}\right)+4 d s_{1}^{0}\left(\mathcal{P} r_{1}\right)+2 d s_{1}^{\prime}\left(\mathcal{P} r_{1}\right)+4 s d_{1}^{0}\left(\mathcal{P} r_{1}\right) \\
& +2 s d_{1}^{\prime}\left(\mathcal{P} r_{1}\right)+4 s s_{1}^{0}\left(\mathcal{P} r_{1}\right)+\overline{d d^{\prime}}{ }_{2}\left(\mathcal{P} r_{1}\right)+\widetilde{d d^{\prime}}{ }_{2}\left(\mathcal{P} r_{1}\right)+4 \overrightarrow{d d^{\prime \prime}}{ }_{2}\left(\mathcal{P} r_{1}\right) \\
& +2 \overleftarrow{d d^{\prime \prime}}{ }_{2}\left(\mathcal{P} r_{1}\right)+2 d s_{2}^{\prime}\left(\mathcal{P} r_{1}\right)+2 s d_{2}^{\prime}\left(\mathcal{P} r_{1}\right)+4 s s_{2}^{1}\left(\mathcal{P} r_{1}\right)+3 s s_{2}^{2}\left(\mathcal{P} r_{1}\right) \\
& +s s_{3}^{2}\left(\mathcal{P} r_{1}\right) \\
& =2 \times 0+2 \times 0+3 \times 0+3 \times 0+4 \times 0+4 \times 0+2 \times 0+4 \times 4+2 \times 8 \\
& +4 \times 4+2 \times 8+4 \times 0+0+0+4 \times 0+2 \times 0+2 \times 0+2 \times 0 \\
& +4 \times 24+3 \times 0+0 \\
& =160
\end{aligned}
$$

$$
\begin{aligned}
g_{0}\left(\mathcal{C P} r_{2}\right)= & {\overline{d d^{\prime}}}_{0}\left(\mathcal{P} r_{2}\right)+\widetilde{d d^{\prime}}{ }_{0}\left(\mathcal{P} r_{2}\right)+4{\overrightarrow{d d^{\prime \prime}}}_{0}\left(\mathcal{P} r_{2}\right)+2 d^{\prime \prime}{ }_{0}\left(\mathcal{P} r_{2}\right)+2 d s_{0}^{\prime}\left(\mathcal{P} r_{2}\right) \\
& +2 s d_{0}^{\prime}\left(\mathcal{P} r_{2}\right)+4 s s_{0}^{1}\left(\mathcal{P} r_{2}\right)+3 s s_{0}^{2}\left(\mathcal{P} r_{2}\right)+s s_{1}^{2}\left(\mathcal{P} r_{2}\right) \\
= & 1+1+4 \times 0+2 \times 0+2 \times 0+2 \times 0+4 \times 0+3 \times 0+0 \\
= & 2
\end{aligned}
$$$$
g_{1}\left(\mathcal{C P} r_{2}\right)=2 d d_{0}^{0}\left(\mathcal{P} r_{2}\right)+3 \overline{d d^{\prime}}{ }_{0}\left(\mathcal{P} r_{2}\right)+3 \widetilde{d d^{\prime}}{ }_{0}\left(\mathcal{P} r_{2}\right)+4{\overrightarrow{d d^{\prime}}}_{0}\left(\mathcal{P} r_{2}\right)+4 \overleftarrow{d d^{\prime}}{ }_{0}\left(\mathcal{P} r_{2}\right)
$$$$
+2 \overleftarrow{d d^{\prime \prime}}{ }_{0}\left(\mathcal{P} r_{2}\right)+4 d s_{0}^{0}\left(\mathcal{P} r_{2}\right)+2 d s_{0}^{\prime}\left(\mathcal{P} r_{2}\right)+4 s d_{0}^{0}\left(\mathcal{P} r_{2}\right)+2 s d_{0}^{\prime}\left(\mathcal{P} r_{2}\right)
$$$$
+4 s s_{0}^{0}\left(\mathcal{P} r_{2}\right)+{\overline{d d^{\prime}}}_{1}\left(\mathcal{P} r_{2}\right)+\widetilde{d d^{\prime}}{ }_{1}\left(\mathcal{P} r_{2}\right)+4{\overrightarrow{d d^{\prime \prime}}}_{1}\left(\mathcal{P} r_{2}\right)+2 \overleftarrow{d d^{\prime \prime}}{ }_{1}\left(\mathcal{P} r_{2}\right)
$$$$
+2 d s_{1}^{\prime}\left(\mathcal{P} r_{2}\right)+2 s d_{1}^{\prime}\left(\mathcal{P} r_{2}\right)+4 s s_{1}^{1}\left(\mathcal{P} r_{2}\right)+3 s s_{1}^{2}\left(\mathcal{P} r_{2}\right)+s s_{2}^{2}\left(\mathcal{P} r_{2}\right)
$$$$
=2 \times 6+3 \times 1+3 \times 1+4 \times 0+4 \times 0+2 \times 0+4 \times 0+2 \times 0+4 \times 0
$$$$
+2 \times 0+4 \times 0+46+46+4 \times 0+2 \times 0+2 \times 12+2 \times 12+4 \times 0
$$$$
+3 \times 0+8
$$$$
=166
$$ 


$$
\begin{aligned}
& g_{2}\left(\mathcal{C P} r_{2}\right)=2 d d_{0}^{0}\left(\mathcal{P} r_{2}\right)+2 d d_{1}^{0}\left(\mathcal{P} r_{2}\right)+3 \overline{d d^{\prime}}{ }_{1}\left(\mathcal{P} r_{2}\right)+3 \widetilde{d d^{\prime}}{ }_{1}\left(\mathcal{P} r_{2}\right)+4 \overrightarrow{d d^{\prime}}{ }_{1}\left(\mathcal{P} r_{2}\right) \\
& +4 \overleftarrow{d d^{\prime}}{ }_{1}\left(\mathcal{P} r_{2}\right)+2 \overleftarrow{d d^{\prime \prime}}{ }_{1}\left(\mathcal{P} r_{2}\right)+4 d s_{1}^{0}\left(\mathcal{P} r_{2}\right)+2 d s_{1}^{\prime}\left(\mathcal{P} r_{2}\right)+4 s d_{1}^{0}\left(\mathcal{P} r_{2}\right) \\
& +2 s d_{1}^{\prime}\left(\mathcal{P} r_{2}\right)+4 s s_{1}^{0}\left(\mathcal{P} r_{2}\right)+{\overline{d d^{\prime}}}_{2}\left(\mathcal{P} r_{2}\right)+\widetilde{d d^{\prime}}{ }_{2}\left(\mathcal{P} r_{2}\right)+4{\overrightarrow{d d^{\prime \prime}}}_{2}\left(\mathcal{P} r_{2}\right) \\
& +2 \overleftarrow{d d^{\prime \prime}}{ }_{2}\left(\mathcal{P} r_{2}\right)+2 d s_{2}^{\prime}\left(\mathcal{P} r_{2}\right)+2 s d_{2}^{\prime}\left(\mathcal{P} r_{2}\right)+4 s s_{2}^{1}\left(\mathcal{P} r_{2}\right)+3 s s_{2}^{2}\left(\mathcal{P} r_{2}\right) \\
& +s s_{3}^{2}\left(\mathcal{P} r_{2}\right) \\
& =2 \times 6+2 \times 176+3 \times 46+3 \times 46+4 \times 22+4 \times 22+2 \times 0+4 \times 44 \\
& +2 \times 12+4 \times 44+2 \times 12+4 \times 0+400+400+4 \times 48+2 \times 48 \\
& +2 \times 544+2 \times 544+4 \times 72+3 \times 8+288 \\
& =5080 \\
& g_{3}\left(\mathcal{C P} r_{2}\right)=2 d d_{1}^{0}\left(\mathcal{P} r_{2}\right)+2 d d_{2}^{0}\left(\mathcal{P} r_{2}\right)+3 \overline{d d^{\prime}}{ }_{2}\left(\mathcal{P} r_{2}\right)+3 \widetilde{d d^{\prime}}{ }_{2}\left(\mathcal{P} r_{2}\right)+4{\overrightarrow{d d^{\prime}}}_{2}\left(\mathcal{P} r_{2}\right) \\
& +4 \overleftarrow{d d^{\prime}}{ }_{2}\left(\mathcal{P} r_{2}\right)+2 \overleftarrow{d d^{\prime \prime}}{ }_{2}\left(\mathcal{P} r_{2}\right)+4 d s_{2}^{0}\left(\mathcal{P} r_{2}\right)+2 d s_{2}^{\prime}\left(\mathcal{P} r_{2}\right)+4 s d_{2}^{0}\left(\mathcal{P} r_{2}\right) \\
& +2 s d_{2}^{\prime}\left(\mathcal{P} r_{2}\right)+4 s s_{2}^{0}\left(\mathcal{P} r_{2}\right)+\overline{d d^{\prime}}{ }_{3}\left(\mathcal{P} r_{2}\right)+{\widetilde{d d^{\prime}}}_{3}\left(\mathcal{P} r_{2}\right)+4{\overrightarrow{d d^{\prime \prime}}}_{3}\left(\mathcal{P} r_{2}\right) \\
& +2 \overleftarrow{d d^{\prime \prime}}{ }_{3}\left(\mathcal{P} r_{2}\right)+2 d s_{3}^{\prime}\left(\mathcal{P} r_{2}\right)+2 s d_{3}^{\prime}\left(\mathcal{P} r_{2}\right)+4 s s_{3}^{1}\left(\mathcal{P} r_{2}\right)+3 s s_{3}^{2}\left(\mathcal{P} r_{2}\right) \\
& +s s_{4}^{2}\left(\mathcal{P} r_{2}\right) \\
& =2 \times 176+2 \times 704+3 \times 400+3 \times 400+4 \times 256+4 \times 256+2 \times 48 \\
& +4 \times 800+2 \times 544+4 \times 800+2 \times 544+4 \times 320+0+0+4 \times 0 \\
& +2 \times 0+2 \times 1920+2 \times 1920+4 \times 1664+3 \times 288+0 \\
& =31360 \\
& g_{4}\left(\mathcal{C P} r_{2}\right)=2 d d_{2}^{0}\left(\mathcal{P} r_{2}\right)+2 d d_{3}^{0}\left(\mathcal{P} r_{2}\right)+3 \overline{d d^{\prime}}{ }_{3}\left(\mathcal{P} r_{2}\right)+3 \widetilde{d d^{\prime}}{ }_{3}\left(\mathcal{P} r_{2}\right)+4{\overrightarrow{d d^{\prime}}}_{3}\left(\mathcal{P} r_{2}\right) \\
& +4 \overleftarrow{d d^{\prime}}{ }_{3}\left(\mathcal{P} r_{2}\right)+2 \overleftarrow{d d^{\prime \prime}}{ }_{3}\left(\mathcal{P} r_{2}\right)+4 d s_{3}^{0}\left(\mathcal{P} r_{2}\right)+2 d s_{3}^{\prime}\left(\mathcal{P} r_{2}\right)+4 s d_{3}^{0}\left(\mathcal{P} r_{2}\right) \\
& +2 s d_{3}^{\prime}\left(\mathcal{P} r_{2}\right)+4 s s_{3}^{0}\left(\mathcal{P} r_{2}\right)+\overline{d d^{\prime}}{ }_{4}\left(\mathcal{P} r_{2}\right)+\widetilde{d d^{\prime}}{ }_{4}\left(\mathcal{P} r_{2}\right)+4 \overrightarrow{d d^{\prime \prime}}{ }_{4}\left(\mathcal{P} r_{2}\right) \\
& +2 \overleftarrow{d d^{\prime \prime}}{ }_{4}\left(\mathcal{P} r_{2}\right)+2 d s_{4}^{\prime}\left(\mathcal{P} r_{2}\right)+2 s d_{4}^{\prime}\left(\mathcal{P} r_{2}\right)+4 s s_{4}^{1}\left(\mathcal{P} r_{2}\right)+3 s s_{4}^{2}\left(\mathcal{P} r_{2}\right) \\
& +s s_{5}^{2}\left(\mathcal{P} r_{2}\right) \\
& =2 \times 704+2 \times 0+3 \times 0+3 \times 0+4 \times 0+4 \times 0+2 \times 0+4 \times 384 \\
& +2 \times 1920+4 \times 384+2 \times 1920+4 \times 1888+0+0+4 \times 0+2 \times 0 \\
& +2 \times 0+2 \times 0+4 \times 2304+3 \times 0+0 \\
& =28928
\end{aligned}
$$

Let $\mathcal{K} \mathcal{P} r_{n}$ be the contra-self-amalgamated closed chain of $n$ copies of $\Delta_{G}$, as illustrated in Figure 15. We calculate genus distributions for contra-self-amalgamated closed chains $\mathcal{K} \mathcal{P} r_{1}$ and $\mathcal{K} \mathcal{P} r_{2}$ by substituting values from Table 2 into Equation (2.22) as follows:

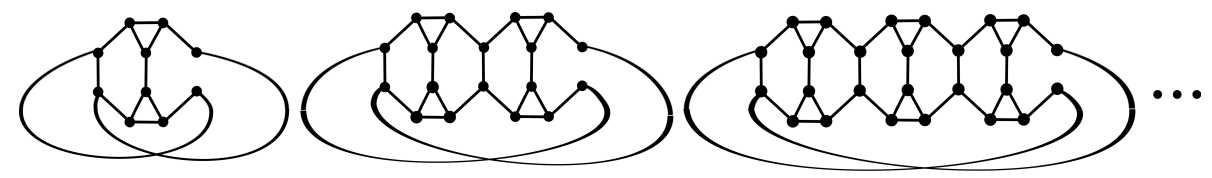

Figure 15: Contra-pasted closed chains of copies of a triangular prism graph. 


$$
\begin{aligned}
g_{0}\left(\mathcal{K} \mathcal{P} r_{1}\right)= & {\overrightarrow{d d^{\prime}}}_{0}\left(\mathcal{P} r_{1}\right)+\overleftarrow{d d^{\prime}}{ }_{0}\left(\mathcal{P} r_{1}\right)+4 \overleftarrow{d d^{\prime \prime}}{ }_{0}\left(\mathcal{P} r_{1}\right)+2{\overrightarrow{d d^{\prime \prime}}}_{0}\left(\mathcal{P} r_{1}\right)+2 d s_{0}^{\prime}\left(\mathcal{P} r_{1}\right) \\
& +2 s d_{0}^{\prime}\left(\mathcal{P} r_{1}\right)+4 s s_{0}^{1}\left(\mathcal{P} r_{1}\right)+3 s s_{0}^{2}\left(\mathcal{P} r_{1}\right)+s s_{1}^{2}\left(\mathcal{P} r_{1}\right) \\
= & 0+0+4 \times 0+2 \times 0+2 \times 0+2 \times 0+4 \times 0+3 \times 0+2 \\
= & 2
\end{aligned}
$$$$
g_{1}\left(\mathcal{K} \mathcal{P} r_{1}\right)=2 d d_{0}^{0}\left(\mathcal{P} r_{1}\right)+3{\overrightarrow{d d^{\prime}}}_{0}\left(\mathcal{P} r_{1}\right)+3 \overleftarrow{d d^{\prime}}{ }_{0}\left(\mathcal{P} r_{1}\right)+4{\overline{d d^{\prime}}}_{0}\left(\mathcal{P} r_{1}\right)+4 \widetilde{d d^{\prime}}{ }_{0}\left(\mathcal{P} r_{1}\right)
$$$$
+2 \overrightarrow{d d^{\prime \prime}}{ }_{0}\left(\mathcal{P} r_{1}\right)+4 d s_{0}^{0}\left(\mathcal{P} r_{1}\right)+2 d s_{0}^{\prime}\left(\mathcal{P} r_{1}\right)+4 s d_{0}^{0}\left(\mathcal{P} r_{1}\right)+2 s d_{0}^{\prime}\left(\mathcal{P} r_{1}\right)
$$$$
+4 s s_{0}^{0}\left(\mathcal{P} r_{1}\right)+{\overrightarrow{d d^{\prime}}}_{1}\left(\mathcal{P} r_{1}\right)+\overleftarrow{d d^{\prime}}{ }_{1}\left(\mathcal{P} r_{1}\right)+4 \overleftarrow{d d^{\prime \prime}}{ }_{1}\left(\mathcal{P} r_{1}\right)+2 \overrightarrow{d d^{\prime \prime}}{ }_{1}\left(\mathcal{P} r_{1}\right)
$$$$
+2 d s_{1}^{\prime}\left(\mathcal{P} r_{1}\right)+2 s d_{1}^{\prime}\left(\mathcal{P} r_{1}\right)+4 s s_{1}^{1}\left(\mathcal{P} r_{1}\right)+3 s s_{1}^{2}\left(\mathcal{P} r_{1}\right)+s s_{2}^{2}\left(\mathcal{P} r_{1}\right)
$$$$
=2 \times 0+3 \times 0+3 \times 0+4 \times 1+4 \times 1+2 \times 0+4 \times 0+2 \times 0+4 \times 0
$$$$
+2 \times 0+4 \times 0+0+0+4 \times 0+2 \times 12+2 \times 8+2 \times 8+4 \times 0+3 \times 2
$$$$
+0
$$

$$
\begin{aligned}
= & 70 \\
g_{2}\left(\mathcal{K} \mathcal{P} r_{1}\right)= & 2 d d_{0}^{0}\left(\mathcal{P} r_{1}\right)+2 d d_{1}^{0}\left(\mathcal{P} r_{1}\right)+3 \overrightarrow{d d^{\prime}}{ }_{1}\left(\mathcal{P} r_{1}\right)+3 \overleftarrow{d d^{\prime}}{ }_{1}\left(\mathcal{P} r_{1}\right)+4{\overrightarrow{d d^{\prime}}}_{1}\left(\mathcal{P} r_{1}\right) \\
& +4{\widetilde{d d^{\prime}}}_{1}\left(\mathcal{P} r_{1}\right)+2 \overrightarrow{d d^{\prime \prime}}{ }_{1}\left(\mathcal{P} r_{1}\right)+4 d s_{1}^{0}\left(\mathcal{P} r_{1}\right)+2 d s_{1}^{\prime}\left(\mathcal{P} r_{1}\right)+4 s d_{1}^{0}\left(\mathcal{P} r_{1}\right) \\
& +2 s d_{1}^{\prime}\left(\mathcal{P} r_{1}\right)+4 s s_{1}^{0}\left(\mathcal{P} r_{1}\right)+{\overrightarrow{d d^{\prime}}}_{2}\left(\mathcal{P} r_{1}\right)+{\overleftarrow{d d^{\prime}}}_{2}\left(\mathcal{P} r_{1}\right)+4{\overleftarrow{d d^{\prime \prime}}}_{2}\left(\mathcal{P} r_{1}\right) \\
& +2{\overrightarrow{d d^{\prime \prime}}}_{2}\left(\mathcal{P} r_{1}\right)+2 d s_{2}^{\prime}\left(\mathcal{P} r_{1}\right)+2 s d_{2}^{\prime}\left(\mathcal{P} r_{1}\right)+4 s s_{2}\left(\mathcal{P} r_{1}\right)+3 s s_{2}^{2}\left(\mathcal{P} r_{1}\right) \\
& +s s_{3}^{2}\left(\mathcal{P} r_{1}\right) \\
= & 2 \times 0+2 \times 0+3 \times 0+3 \times 0+4 \times 0+4 \times 0+2 \times 12+4 \times 4+2 \times 8 \\
& +4 \times 4+2 \times 8+4 \times 0+0+0+4 \times 0+2 \times 0+2 \times 0+2 \times 0 \\
& +4 \times 24+3 \times 0+0 \\
= & 184
\end{aligned}
$$

$$
\begin{aligned}
g_{0}\left(\mathcal{K} \mathcal{P} r_{2}\right)= & {\overrightarrow{d d^{\prime}}}_{0}\left(\mathcal{P} r_{2}\right)+\overleftarrow{d d^{\prime}}{ }_{0}\left(\mathcal{P} r_{2}\right)+4 \overleftarrow{d d^{\prime \prime}}{ }_{0}\left(\mathcal{P} r_{2}\right)+2{\overrightarrow{d d^{\prime \prime}}}_{0}\left(\mathcal{P} r_{2}\right)+2 d s_{0}^{\prime}\left(\mathcal{P} r_{2}\right) \\
& +2 s d_{0}^{\prime}\left(\mathcal{P} r_{2}\right)+4 s s_{0}^{1}\left(\mathcal{P} r_{2}\right)+3 s s_{0}^{2}\left(\mathcal{P} r_{2}\right)+s s_{1}^{2}\left(\mathcal{P} r_{2}\right) \\
= & 0+0+4 \times 0+2 \times 0+2 \times 0+2 \times 0+4 \times 0+3 \times 0+0 \\
= & 0
\end{aligned}
$$$$
g_{1}\left(\mathcal{K} \mathcal{P} r_{2}\right)=2 d d_{0}^{0}\left(\mathcal{P} r_{2}\right)+3{\overrightarrow{d d^{\prime}}}_{0}\left(\mathcal{P} r_{2}\right)+3 \overleftarrow{d d^{\prime}}{ }_{0}\left(\mathcal{P} r_{2}\right)+4{\overline{d d^{\prime}}}_{0}\left(\mathcal{P} r_{2}\right)+4 \widetilde{d d^{\prime}}{ }_{0}\left(\mathcal{P} r_{2}\right)
$$$$
+2{\overrightarrow{d d^{\prime \prime}}}_{0}\left(\mathcal{P} r_{2}\right)+4 d s_{0}^{0}\left(\mathcal{P} r_{2}\right)+2 d s_{0}^{\prime}\left(\mathcal{P} r_{2}\right)+4 s d_{0}^{0}\left(\mathcal{P} r_{2}\right)+2 s d_{0}^{\prime}\left(\mathcal{P} r_{2}\right)
$$$$
+4 s s_{0}^{0}\left(\mathcal{P} r_{2}\right)+{\overrightarrow{d d^{\prime}}}_{1}\left(\mathcal{P} r_{2}\right)+\overleftarrow{d d^{\prime}}{ }_{1}\left(\mathcal{P} r_{2}\right)+4 \overleftarrow{d d^{\prime \prime}}{ }_{1}\left(\mathcal{P} r_{2}\right)+2 \overrightarrow{d d^{\prime \prime}}{ }_{1}\left(\mathcal{P} r_{2}\right)
$$$$
+2 d s_{1}^{\prime}\left(\mathcal{P} r_{2}\right)+2 s d_{1}^{\prime}\left(\mathcal{P} r_{2}\right)+4 s s_{1}^{1}\left(\mathcal{P} r_{2}\right)+3 s s_{1}^{2}\left(\mathcal{P} r_{2}\right)+s s_{2}^{2}\left(\mathcal{P} r_{2}\right)
$$$$
=2 \times 6+3 \times 0+3 \times 0+4 \times 1+4 \times 1+2 \times 0+4 \times 0+2 \times 0+4 \times 0
$$$$
+2 \times 0+4 \times 0+22+22+4 \times 0+2 \times 0+2 \times 12+2 \times 12+4 \times 0
$$$$
+3 \times 0+8
$$$$
=120
$$ 


$$
\begin{aligned}
& g_{2}\left(\mathcal{K} \mathcal{P} r_{2}\right)=2 d d_{0}^{0}\left(\mathcal{P} r_{2}\right)+2 d d_{1}^{0}\left(\mathcal{P} r_{2}\right)+3{\overrightarrow{d d^{\prime}}}_{1}\left(\mathcal{P} r_{2}\right)+3 \overleftarrow{d d^{\prime}}{ }_{1}\left(\mathcal{P} r_{2}\right)+4 \overline{d d^{\prime}}{ }_{1}\left(\mathcal{P} r_{2}\right) \\
& +4 \widetilde{d d^{\prime}}{ }_{1}\left(\mathcal{P} r_{2}\right)+2 \overrightarrow{d d^{\prime \prime}}{ }_{1}\left(\mathcal{P} r_{2}\right)+4 d s_{1}^{0}\left(\mathcal{P} r_{2}\right)+2 d s_{1}^{\prime}\left(\mathcal{P} r_{2}\right)+4 s d_{1}^{0}\left(\mathcal{P} r_{2}\right) \\
& +2 s d_{1}^{\prime}\left(\mathcal{P} r_{2}\right)+4 s s_{1}^{0}\left(\mathcal{P} r_{2}\right)+{\overrightarrow{d d^{\prime}}}_{2}\left(\mathcal{P} r_{2}\right)+\overleftarrow{d d^{\prime}}{ }_{2}\left(\mathcal{P} r_{2}\right)+4 \overleftarrow{d d^{\prime \prime}}{ }_{2}\left(\mathcal{P} r_{2}\right) \\
& +2 \overrightarrow{d d^{\prime \prime}}{ }_{2}\left(\mathcal{P} r_{2}\right)+2 d s_{2}^{\prime}\left(\mathcal{P} r_{2}\right)+2 s d_{2}^{\prime}\left(\mathcal{P} r_{2}\right)+4 s s_{2}^{1}\left(\mathcal{P} r_{2}\right)+3 s s_{2}^{2}\left(\mathcal{P} r_{2}\right) \\
& +s s_{3}^{2}\left(\mathcal{P} r_{2}\right) \\
& =2 \times 6+2 \times 176+3 \times 22+3 \times 22+4 \times 46+4 \times 46+2 \times 0+4 \times 44 \\
& +2 \times 12+4 \times 44+2 \times 12+4 \times 0+256+256+4 \times 48+2 \times 48 \\
& +2 \times 544+2 \times 544+4 \times 72+3 \times 8+288 \\
& =4840 \\
& g_{3}\left(\mathcal{K} \mathcal{P} r_{2}\right)=2 d d_{1}^{0}\left(\mathcal{P} r_{2}\right)+2 d d_{2}^{0}\left(\mathcal{P} r_{2}\right)+3{\overrightarrow{d d^{\prime}}}_{2}\left(\mathcal{P} r_{2}\right)+3 \overleftarrow{d d^{\prime}}{ }_{2}\left(\mathcal{P} r_{2}\right)+4 \overline{d d^{\prime}}{ }_{2}\left(\mathcal{P} r_{2}\right) \\
& +4 \widetilde{d d^{\prime}}{ }_{2}\left(\mathcal{P} r_{2}\right)+2 \overrightarrow{d d^{\prime \prime}}{ }_{2}\left(\mathcal{P} r_{2}\right)+4 d s_{2}^{0}\left(\mathcal{P} r_{2}\right)+2 d s_{2}^{\prime}\left(\mathcal{P} r_{2}\right)+4 s d_{2}^{0}\left(\mathcal{P} r_{2}\right) \\
& +2 s d_{2}^{\prime}\left(\mathcal{P} r_{2}\right)+4 s s_{2}^{0}\left(\mathcal{P} r_{2}\right)+{\overrightarrow{d d^{\prime}}}_{3}\left(\mathcal{P} r_{2}\right)+\overleftarrow{d d^{\prime}}{ }_{3}\left(\mathcal{P} r_{2}\right)+4 \overleftarrow{d d^{\prime \prime}}{ }_{3}\left(\mathcal{P} r_{2}\right) \\
& +2 \overrightarrow{d d^{\prime \prime}}{ }_{3}\left(\mathcal{P} r_{2}\right)+2 d s_{3}^{\prime}\left(\mathcal{P} r_{2}\right)+2 s d_{3}^{\prime}\left(\mathcal{P} r_{2}\right)+4 s s_{3}^{1}\left(\mathcal{P} r_{2}\right)+3 s s_{3}^{2}\left(\mathcal{P} r_{2}\right) \\
& +s s_{4}^{2}\left(\mathcal{P} r_{2}\right) \\
& =2 \times 176+2 \times 704+3 \times 256+3 \times 256+4 \times 400+4 \times 400+2 \times 48 \\
& +4 \times 800+2 \times 544+4 \times 800+2 \times 544+4 \times 320+0+0+4 \times 0 \\
& +2 \times 0+2 \times 1920+2 \times 1920+4 \times 1664+3 \times 288+0 \\
& =31648 \\
& g_{4}\left(\mathcal{K} \mathcal{P} r_{2}\right)=2 d d_{2}^{0}\left(\mathcal{P} r_{2}\right)+2 d d_{3}^{0}\left(\mathcal{P} r_{2}\right)+3{\overrightarrow{d d^{\prime}}}_{3}\left(\mathcal{P} r_{2}\right)+3 \overleftarrow{d d^{\prime}}{ }_{3}\left(\mathcal{P} r_{2}\right)+4 \overline{d d^{\prime}}{ }_{3}\left(\mathcal{P} r_{2}\right) \\
& +4 \widetilde{d d^{\prime}}{ }_{3}\left(\mathcal{P} r_{2}\right)+2 \overrightarrow{d d^{\prime \prime}}{ }_{3}\left(\mathcal{P} r_{2}\right)+4 d s_{3}^{0}\left(\mathcal{P} r_{2}\right)+2 d s_{3}^{\prime}\left(\mathcal{P} r_{2}\right)+4 s d_{3}^{0}\left(\mathcal{P} r_{2}\right) \\
& +2 s d_{3}^{\prime}\left(\mathcal{P} r_{2}\right)+4 s s_{3}^{0}\left(\mathcal{P} r_{2}\right)+{\overrightarrow{d d^{\prime}}}_{4}\left(\mathcal{P} r_{2}\right)+\overleftarrow{d d^{\prime}}{ }_{4}\left(\mathcal{P} r_{2}\right)+4 \overleftarrow{d d^{\prime \prime}}{ }_{4}\left(\mathcal{P} r_{2}\right) \\
& +2 \overrightarrow{d d^{\prime \prime}}{ }_{4}\left(\mathcal{P} r_{2}\right)+2 d s_{4}^{\prime}\left(\mathcal{P} r_{2}\right)+2 s d_{4}^{\prime}\left(\mathcal{P} r_{2}\right)+4 s s_{4}^{1}\left(\mathcal{P} r_{2}\right)+3 s s_{4}^{2}\left(\mathcal{P} r_{2}\right) \\
& +s s_{5}^{2}\left(\mathcal{P} r_{2}\right) \\
& =2 \times 704+2 \times 0+3 \times 0+3 \times 0+4 \times 0+4 \times 0+2 \times 0+4 \times 384 \\
& +2 \times 1920+4 \times 384+2 \times 1920+4 \times 1888+0+0+4 \times 0 \\
& +2 \times 0+2 \times 0+2 \times 0+4 \times 2304+3 \times 0+0 \\
& =28928
\end{aligned}
$$

In a similar manner, we can compute the genus distributions for $\mathcal{C P} r_{n}$ and $\mathcal{K} \mathcal{P} r_{n}$ for higher values of $n$. We conclude this section by giving the partitioned genus distribution of $\mathcal{P} r_{3}$ in Table 3 and listing the genus distributions of $\mathcal{C} \mathcal{P} r_{3}$ and $\mathcal{K} \mathcal{P} r_{3}$ obtained similarly. 
Table 3: Genus distributions of the open chain $\mathcal{P} r_{3}$ of 3 copies of $\Delta_{G}$.

\begin{tabular}{|c|rrrrrrr|}
\hline \hline $\mathcal{P} r_{n}$ & \multicolumn{7}{|c}{$\mathcal{P} r_{3}$} \\
\hline$k$ & 0 & 1 & 2 & 3 & 4 & 5 & 6 \\
\hline$d d_{k}^{0}$ & 30 & 2080 & 40000 & 211840 & 67584 & 0 & 0 \\
${\overrightarrow{d d^{\prime}}}_{k}$ & 1 & 82 & 2608 & 27104 & 38400 & 0 & 0 \\
${\widetilde{d d^{\prime}}}_{k}$ & 1 & 82 & 2608 & 27104 & 38400 & 0 & 0 \\
${\overrightarrow{d d^{\prime}}}_{k}$ & 0 & 46 & 2176 & 25376 & 38400 & 0 & 0 \\
${\overleftarrow{d d^{\prime}}}_{k}$ & 0 & 46 & 2176 & 25376 & 38400 & 0 & 0 \\
${\overrightarrow{d d^{\prime \prime}}}_{k}$ & 0 & 0 & 0 & 288 & 3456 & 0 & 0 \\
$\overleftarrow{d d^{\prime \prime}}{ }_{k}$ & 0 & 0 & 0 & 288 & 3456 & 0 & 0 \\
$d s_{k}^{0}$ & 0 & 212 & 11744 & 149568 & 336896 & 36864 & 0 \\
${\overrightarrow{d s^{\prime}}}_{k}$ & 0 & 6 & 640 & 17664 & 113792 & 92160 & 0 \\
$\overleftarrow{d s^{\prime}}{ }_{k}$ & 0 & 6 & 640 & 17664 & 113792 & 92160 & 0 \\
$s d_{k}^{0}$ & 0 & 212 & 11744 & 149568 & 336896 & 36864 & 0 \\
${\overrightarrow{s d^{\prime}}}_{k}$ & 0 & 6 & 640 & 17664 & 113792 & 92160 & 0 \\
$\overleftarrow{s d^{\prime}}{ }_{k}$ & 0 & 6 & 640 & 17664 & 113792 & 92160 & 0 \\
$s s_{k}^{0}$ & 0 & 0 & 1496 & 61728 & 393728 & 417792 & 0 \\
$s s_{k}^{1}$ & 0 & 0 & 72 & 6144 & 112000 & 411648 & 221184 \\
$s s_{k}^{2}$ & 0 & 0 & 0 & 32 & 3456 & 0 & 0 \\
\hline$g_{k}$ & 32 & 2784 & 77184 & 755072 & 1866240 & 1271808 & 221184 \\
\hline \hline
\end{tabular}

$$
\begin{aligned}
& g_{0}\left(\mathcal{C P} r_{3}\right)=2 \\
& g_{1}\left(\mathcal{C P} r_{3}\right)=278 \\
& g_{2}\left(\mathcal{C P} r_{3}\right)=17480 \\
& g_{3}\left(\mathcal{C P} r_{3}\right)=447648 \\
& g_{4}\left(\mathcal{C P} r_{3}\right)=3920896 \\
& g_{5}\left(\mathcal{C P} r_{3}\right)=8667648 \\
& g_{6}\left(\mathcal{C P} r_{3}\right)=3723264 \\
& g_{0}\left(\mathcal{K} \mathcal{P} r_{3}\right)=0 \\
& g_{1}\left(\mathcal{K} \mathcal{P} r_{3}\right)=208 \\
& g_{2}\left(\mathcal{K} \mathcal{P} r_{3}\right)=16688 \\
& g_{3}\left(\mathcal{K} \mathcal{P} r_{3}\right)=445056 \\
& g_{4}\left(\mathcal{K} \mathcal{P} r_{3}\right)=3924352 \\
& g_{5}\left(\mathcal{K} \mathcal{P} r_{3}\right)=8667648 \\
& g_{6}\left(\mathcal{K} \mathcal{P} r_{3}\right)=3723264
\end{aligned}
$$




\section{Conclusions}

This paper derives closed formulas for calculating the genus distributions of graphs resulting from co-self-amalgamation and contra-self-amalgamation of any double-edge-rooted graph $(G, e, f)$, where both the edges $e$ and $f$ have two 2-valent endpoints and the doubleroot partitioned genus distribution of $(G, e, f)$ is known. This enables one to make genus distribution calculations for edge-amalgamated closed chains corresponding to many infinite families of open chains. To illustrate this, we demonstrated how the genus distributions of the circular ladder $C L_{4}$ and the Möbius ladder $M L_{4}$ can be calculated from the partitioned genus distribution of the closed-end ladder $L_{3}$. We also showed genus distribution calculations of small co-self-pasted and contra-self-pasted closed chains of copies of the triangular prism graph. In this manner, calculating genus distribution of arbitrarily large closed chains consisting of identical copies of the same graph as well as well interleaved copies of different graphs becomes tractible.

It would be interesting to know if open as well as closed chains of base graphs with strongly unimodal genus distributions are unimodal. The results in this paper formulate the genus distribution of closed chains as a linear combination of sub-partials of the corresponding open chains. In turn, each of these sub-partials of an open chain is calculated as a linear combination of convolutions of sub-partials of its constituent subgraphs. Although convolutions of strongly unimodal sequences are strongly unimodal, linear combinations of unimodal sequences need not be unimodal, unless the modes are sufficiently close together. This seems quite complicated, due to the large number of sub-partials. Nevertheless, the analysis of the closed-formulas presented in this paper may prove to be useful for establishing structural results related to genus distributions such as the unimodality conjecture. In particular, if the unimodality conjecture is false, the results could help construct a counterexample to disprove the conjecture. Further opportunities for research also include self-edge-amalgamation on root-edges with endpoints of higher-valence.

\section{References}

[1] C. P. Bonnington, M. J. Grannell, T. S. Griggs, and J. Širáň, Exponential families of nonisomorphic triangulations of complete graphs, J. Combin. Theory (B) 78 (2000), 169-184.

[2] J. Chen, J. L. Gross and R. G. Rieper, Overlap matrices and total imbedding distributions, Discrete Math. 128 (1994), 73-94.

[3] Y. C. Chen, Y. P. Liu and T. Wang, The total embedding distributions of cacti and necklaces, Acta Math. Sinica — English Series 22 (2006), 1583-1590.

[4] M. L. Furst, J. L. Gross and R. Statman, Genus distribution for two classes of graphs, J. Combin. Theory B 46 (1989), 22-36.

[5] L. Goddyn, R. B. Richter and J. Širáň, Triangular embeddings of complete graphs from graceful labellings of paths, J. Combin. Theory (B) 97 (2007), 964-970.

[6] M. J. Grannell and T. S. Griggs, A lower bound for the number of triangular embeddings of some complete graphs and complete regular tripartite graphs, J. Combin. Theory B 98 (2008), 637-650.

[7] J. L. Gross, Genus distribution of graphs under surgery: Adding edges and splitting vertices, New York J. Math. 16 (2010), 161-178.

[8] J. L. Gross, Genus distribution of graph amalgamations: Self-pasting at root-vertices, Australas. J. Combin. 49 (2011), 19-38. 
[9] J. L. Gross, Genus distributions of cubic outerplanar graphs, J. Graph. Algorithms Appl. 15 (2011), 295-316.

[10] J. L. Gross and M. L. Furst, Hierarchy for imbedding-distribution invariants of a graph, J. Graph Theory 11 (1987), 205-220.

[11] J. L. Gross, I. F. Khan and M. I. Poshni, Genus distribution of graph amalgamations: Pasting at root-vertices, Ars Combin. 94 (2010), 33-53.

[12] J. L. Gross, D. P. Robbins and T. W. Tucker, Genus distributions for bouquets of circles, J. Combin. Theory B 47 (1989), 292-306.

[13] J. L. Gross and T. W. Tucker, Topological Graph Theory, Dover, 2001; (original edn. Wiley, 1987).

[14] I. F. Khan, M. I. Poshni and J. L. Gross, Genus distribution of graph amalgamations: Pasting when one root has arbitrary degree, Ars Math. Contemp. 3 (2010), 121-138.

[15] V. P. Korzhik and H.-J. Voss, Exponential families of non-isomorphic non-triangular orientable genus embeddings of complete graphs, J. Combin. Theory B 86 (2002), 86-211.

[16] J. H. Kwak and J. Lee, Genus polynomials of dipoles, Kyungpook Math. J. 33 (1993), 115-125.

[17] J. H. Kwak and J. Lee, Enumeration of graph embeddings, Discrete Math. 135 (1994), 129151.

[18] J. H. Kwak and S. H. Shim, Total embedding distributions for bouquets of circles, Discrete Math. 248 (2002), 93-108.

[19] L. A. McGeoch, Genus distribution for circular and Möbius ladders, technical report extracted from PhD thesis, Carnegie-Mellon University, 1987.

[20] L. A. McGeoch, Algorithms for two graph problems: computing maximum-genus imbedding and the two-server problem, $\mathrm{PhD}$ thesis, Carnegie-Mellon University, 1987.

[21] B. P. Mull, Enumerating the orientable 2-cell imbeddings of complete bipartite graphs, J. Graph Theory 30 (1999), 77-90.

[22] M. I. Poshni, I. F. Khan, and J. L. Gross, Genus distribution of graphs under edgeamalgamations, Ars Math. Contemp. 3 (2010), 69-86.

[23] M. I. Poshni, I. F. Khan, and J. L. Gross, Genus distributions of 4-regular outerplanar graphs, Electon. J. Comb. 18 (2011), \#P212.

[24] S. Stahl, Region distributions of graph embeddings and Stirling numbers, Discrete Math. 82 (1990), 57-78.

[25] S. Stahl, Permutation-partition pairs III: Embedding distributions of linear families of graphs, J. Combin. Theory B 52 (1991), 191-218.

[26] S. Stahl, Region distributions of some small diameter graphs, Discrete Math. 89 (1991), 281299.

[27] E. H. Tesar, Genus distribution of Ringel ladders, Discrete Math. 216 (2000) 235-252.

[28] T. I. Visentin and S. W. Wieler, On the genus distribution of $(p, q, n)$-dipoles, Electronic J. Combin. 14 (2007), R12.

[29] L. X. Wan and Y. P. Liu, Orientable embedding distributions by genus for certain types of graphs, Ars Combin. 79 (2006), 97-105.

[30] L. X. Wan and Y. P. Liu, Orientable embedding genus distribution for certain types of graphs, J. Combin. Theory B 47 (2008), 19-32.

[31] A. T. White, Graphs of Groups on Surfaces, North-Holland, 2001. 